THU-93/15

ITP-SB 93-353

BRX-TH-349

\title{
THE WZNW MODEL AT TWO LOOPS
}

\author{
B. DE WIT \\ Institute for Theoretical Physics \\ Utrecht University \\ Princetonplein 5, 3508 TA Utrecht, The Netherlands \\ M.T. GRISARUA \\ Department of Physics \\ Brandeis University \\ Waltham, MA 02254, USA \\ and \\ P. VAN NIEUWENHUIZENI \\ Institute for Theoretical Physics \\ State University of New York at Stony Brook \\ Stony Brook, NY 11794-3840, USA
}

\begin{abstract}
We study perturbatively the (conformal) WZNW model. At one loop we compute one-particle irreducible two- and three-point current correlation functions, both in the conventional version and in the classically equivalent, chiral, nonlocal, induced version of the model. At two loops we compute the two-point function and find that it vanishes (modulo infrared-induced logarithms). We use dimensional regularization and the $R^{*}$ operation for removing infrared divergences. The outcome of the calculations is insensitive to the treatment of the $\varepsilon^{\mu \nu}$ tensor as a two-dimensional or $d$-dimensional object. Our results indicate that the one-particle irreducible current correlation functions constitute an effective action equal to the original WZNW action with the familiar level shift, $k \rightarrow k+\tilde{h}$.
\end{abstract}

June 1993

${ }^{*}$ Work partially supported by the National Science Foundation under grant PHY-88-18853.

${ }^{\dagger}$ Work partially supported by the National Science Foundation under grant PHY-92-11367. 


\section{Introduction}

During the past decade the WZNW model [1, 2, 3] has attracted considerable attention, both as a nonlinear sigma model with a nontrivial fixed point [3, 4, 5] and, at the fixed point, a scale invariant model which can be studied with the methods of conformal field theory and current algebra. In particular, the latter approach has led to statements about exact (nonperturbative) properties of the model. Thus, Knizhnik and Zamolodchikov [6] have shown that the existence of exactly conserved spin-1 currents (both at the classical and quantum level) forming a Kac-Moody algebra $\hat{g}$, together with the Virasoro algebra, leads to a determination of the correlation functions of the model.

In its original formulation the WZNW model is described as an ordinary lagrangian field theory. In this formulation, the coupling constant $k$ is restricted to integer values in order that the exponent of the action be single-valued. Already in Witten's work [3] it was argued that the only allowed renormalization of $k$ would be a shift by some integer, and that this could only happen at the one-loop level. Indeed, in their exact algebraic approach, Knizhnik and Zamolodchikov have demonstrated the occurence of a renormalization, or level shift, $k \rightarrow k+\tilde{h}$, where $\tilde{h}$ is the dual Coxeter number of the Lie algebra $g$ associated with the model. In recent work, Leutwyler and Shifman [8] offered explanations of this shift at the level of lagrangian field theory, while at the same time confirming earlier arguments [3], 7] that the current correlation functions coincide with the classical expressions. Tseytlin, studying the effective action, has argued that the shift can be seen to arise, as a one-loop effect, from a functional Jacobian determinant [9]. The implication of this and earlier work is that modulo the shift in $k$ the complete effective action of the WZNW theory is given by the classical action. However, some of the derivations are somewhat formal and make use of the Polyakov-Wiegmann formula [7] and the invariance of the Haar measure without addressing the issue of regularization.

In its usual incarnation the WZNW model is formulated in terms of a scalar field $g(x)$ which takes values in a Lie group $G$. An alternative description is by means of the chiral component $A_{+}(x)$ of a Yang-Mills gauge field [7, 10] and a corresponding non-local, so-called "induced" action [11]. The two versions are connected through the identification $A_{+}=\partial_{+} g g^{-1}$. This induced Yang-Mills action has been used to obtain the induced actions for gravity and $W$-gravity [12, 11, 13], and supergravity [14, 15], as constrained WZNW models. Again $k$ appears as the coupling constant of the model, but now the reasons for its integrality and absence of corrections beyond one loop are less clear. Nonetheless, a variety of arguments have led to conjectures that again the complete effective action is given by the classical action up to a shift in $k$ and a possible multiplicative renormalization $A_{+} \rightarrow Z_{A} A_{+}$. Needless to say, although the two descriptions are identical at the classical level it is by no means certain that their quantum properties will continue to coincide. Besides, while most arguments and calculations agree on a level shift $k \rightarrow k-2 \tilde{h}$, there exists a variety of answers for the factor $Z_{A}[10,17,13,18,15]$.

As a field theory the induced action is not of the standard type. It consists of an infinite series of terms containing inverse derivatives. At first sight the theory seems 
renormalizable since no dimensionful coupling constant is present. However, in loop contributions where inverse derivatives act on external lines the superficial degree of divergence can increase indefinitely. Although invoking symmetries (e.g. Lorentz invariance $\leftrightarrow$ symmetric integration) renders the actual contributions finite [19, 20], the results are in principle ambiguous since one is dealing with superficially linear or higher-order divergent integrals. The relation of the quantum induced action to the quantum WZNW action becomes rather unclear.

In view of the situation described above we decided to embark on an explicit calculation of two-loop effects in the WZNW model, as well as some (one-loop) calculations in the nonlocal induced action. In undertaking this work we were aware of the possibility of stumbling on a vipers' nest, in view of the history of similar two-loop work on the level shift of the three-dimensional Chern-Simons theory [21]. However, we felt that explicit two-loop calculations in the WZNW model beyond

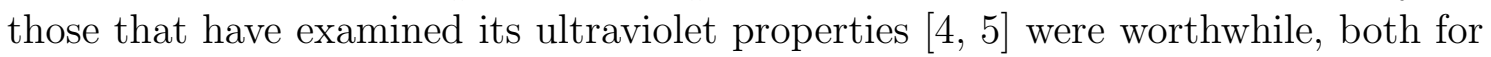
technical reasons and in order to examine problems that arise in applying ordinary quantum field theory techniques to a conformally invariant system. By the same token, the nonlocal induced action represents an interesting arena for examining the application of conventional techniques.

In this paper we present a two-loop calculation of the complete one-particle irreducible two-point function of the WZNW model in a background-field formulation. We start from a covariant background-field action that takes the form of the gauged WZNW model [22], which coincides (at the critical point) with the usual background effective action [3, 团 after truncating to one of the chiral sectors. Because of gauge invariance we do not encounter ultraviolet infinities (except, subtly, in the form of "evanescent" - in dimensional regularization - divergences [4]). Since we use dimensional regularization we have to face the issue of the $\varepsilon^{\mu \nu}$ tensors. Interestingly enough, we find that treating them as $d$-dimensional or, as preferred by sigma-model practitioners [4, 国, two-dimensional tensors, makes no difference to the final answer. However, we are faced with infrared divergences and for these, in ordinary field theory on $\mathbf{R}_{2}$, there is no cure. The best we can do is separate them from what we believe to be the relevant contribution, and show that the latter indeed vanishes at the two-loop level. When combined with the one-loop results (which we also determine here) we thus verify that the effective action, consisting of the sum of the one-particle irreducible current correlation functions, is renormalized by the same level shift $k \rightarrow k+\tilde{h}$ that one encounters in conformal field theory. It is tempting to identify our effective action with the one used by Tseytlin to relate gauged WZNW theories to two-dimensional nonlinear sigma models with a natural target-space interpretation [9]. A more rigorous analysis is clearly hampered by the presence of the infrared divergences. Conceivably, summing the infrared-induced logarithms to all orders of perturbation theory would lead to their complete cancellation, but this issue is beyond the scope of this work.

We also compute at the one-loop level the one-particle irreducible two- and threepoint function in the induced chiral action. Here we use the exponential regularization scheme of Polyakov [19], inserting an exponential damping factor in the loop integral. This scheme has previously been used for the one-loop two-point functions 
of induced Yang-Mills theory [19], two-dimensional gravity [23, 20] and $W_{3}$ gravity [20]. As in these cases, our results are finite and in agreement with those obtained from the semi-classical approximation [10, 16]. Recently similar calculations based on Pauli-Villars regularization were presented in [18.

Our paper is organized as follows: In section 2 we review the WZNW model, both in its local and nonlocal versions. In section 3 we define our (background field) quantization procedure and describe the relation of the resulting action to that of the gauged WZNW model [22]. In section 4, for completeness and in order to set the notation we perform some one-loop computations in the WZNW model. We evaluate the one-particle irreducible two-point function, first by conventional methods, and then it, as well as the three-point function, by the Källén-Toll method [24]. The calculation of the three-point function allows us to verify that the one-loop effective action, defined as the sum of the one-particle irreducible current correlation

functions, takes the form of the WZNW action with the level $k$ shifted to $k+\tilde{h}$ (up to the logarithmic corrections mentioned above). Furthermore we verify that the connected (not one-particle irreducible) current correlation functions remain finite. At the critical point they are not renormalized, as in conformal field theory.

In section 5 we describe one-loop two- and three-point function calculations in the induced action, confirming the level shift $k \rightarrow k-2 \tilde{h}$. Finally, in section 6 we engage in the main task of this paper, the calculation of the complete two-loop, two-point function of the WZNW model. We consider separately contributions from diagrams without and with $\varepsilon^{\mu \nu}$ tensors, and evaluate everything explicitely in dimensional regularization. Along the way we are able to control the calculation by means of the gauge invariance of the original background field action. In the same section we consider the effect of evanescent one-loop counterterms and assemble the final result. As mentioned above, aside from infrared-induced logarithmic terms the two-loop contribution is zero. Section 7 contains our conclusions.

An appendix contains some useful integrals, as well as a discussion of the subtraction techniques we use throughout for handling infrared divergences.

\section{The classical WZNW action and the induced action}

We start by summarizing a number of well-known features of the WZNW action and establish our notation. Later in this section we derive its relation with the action induced by chiral matter coupled to gauge fields associated with a group $G$.

The WZNW action is constructed from two terms, written as an integral over a closed two-dimensional surface $\partial B$ and as an integral over the enclosed volume $B$, respectively,

$$
I[g]=\frac{1}{16 \pi \chi} \int_{\partial B} \mathrm{~d}^{2} x \operatorname{Tr} \partial_{\mu} g g^{-1} \partial^{\mu} g g^{-1}
$$




$$
\Gamma[g]=\frac{1}{24 \pi \chi} \int_{B} \mathrm{~d}^{3} x \varepsilon^{\mu \nu \rho} \operatorname{Tr} \partial_{\mu} g g^{-1} \partial_{\nu} g g^{-1} \partial_{\rho} g g^{-1},
$$

where the fields $g(x)$ take their values in the group $G$. For a compact group the Euclidean action $I[g]$ is thus negative. The normalization of $\Gamma[g]$ is such that, for a compact group, it is well defined modulo an integer times $2 \pi$. Furthermore we note that $I\left[g^{-1}\right]=I[g]$ and $\Gamma\left[g^{-1}\right]=-\Gamma[g]$. We wrote the group elements and the corresponding trace in an irreducible representation of $G$ with generators $T_{a}$. Structure constants are defined by $\left[T_{a}, T_{b}\right]=f_{a b}{ }^{c} T_{c}$. The index $\chi$ of this representation is given by $\operatorname{Tr} T_{a} T_{b}=-\chi g_{a b}$, where $g_{a b}$ denotes the Cartan-Killing metric, defined by $f_{a c}{ }^{d} f_{b d}{ }^{c}=-\tilde{h} g_{a b}$ with $\tilde{h}$ the dual Coxeter number. For instance, for $S U(n)$ we have $\tilde{h}=n$ and, for the fundamental representation, $\chi=\frac{1}{2}$; for $S O(n)$ with $n>4$, these numbers are $n-2$ and 1 , respectively. In the adjoint representation we always have $\chi=\tilde{h}$. We will repeatedly make use of the group theory result $f_{\text {ade }} f_{b e f} f_{c f d}=\frac{1}{2} \tilde{h} f_{a b c}$.

A central role is played by the following multiplication rules,

$$
\begin{aligned}
I[g h] & =I[g]+I[h]+\frac{1}{8 \pi \chi} \int_{\partial B} \mathrm{~d}^{2} x \operatorname{Tr} \partial_{\mu} h h^{-1} g^{-1} \partial^{\mu} g, \\
\Gamma[g h] & =\Gamma[g]+\Gamma[h]+\frac{1}{8 \pi \chi} \int_{\partial B} \mathrm{~d}^{2} x \varepsilon^{\mu \nu} \operatorname{Tr} \partial_{\mu} h h^{-1} g^{-1} \partial_{\nu} g .
\end{aligned}
$$

Parametrizing $g=\exp \pi^{a} T_{a}$, the actions $I[g]$ and $\Gamma[g]$ acquire the form (see, e.g. the first work of [团, eq. (8))

$$
\begin{aligned}
I g] & =-\frac{1}{8 \pi} \int \mathrm{d}^{2} x \sum_{n=1}^{\infty} \frac{1}{(2 n) !} \partial_{\mu} \vec{\pi} \cdot\left(\vec{\pi} \times\left(\vec{\pi} \times \cdots \partial^{\mu} \vec{\pi}\right)\right) \\
& =-\frac{1}{16 \pi} \int \mathrm{d}^{2} x\left\{\left(\partial_{\mu} \vec{\pi}\right)^{2}-\frac{1}{12}\left(\vec{\pi} \times \partial_{\mu} \vec{\pi}\right)^{2}+\mathcal{O}\left(\pi^{6}\right)\right\}, \\
\Gamma[g] & =\frac{1}{8 \pi} \int \mathrm{d}^{2} x \sum_{n=1}^{\infty} \frac{1}{(2 n+1) !} \varepsilon^{\mu \nu} \partial_{\mu} \vec{\pi} \cdot\left(\vec{\pi} \times\left(\vec{\pi} \times \cdots \partial_{\nu} \vec{\pi}\right)\right) \\
& =-\frac{1}{48 \pi} \int \mathrm{d}^{2} x\left\{\varepsilon^{\mu \nu} \vec{\pi} \cdot\left(\partial_{\mu} \vec{\pi} \times \partial_{\nu} \vec{\pi}\right)+\mathcal{O}\left(\pi^{5}\right)\right\},
\end{aligned}
$$

where we used the notation

$$
\vec{x} \cdot \vec{y} \equiv g_{a b} x^{a} y^{b}, \quad(\vec{x} \times \vec{y})^{a} \equiv f_{b c}{ }^{a} x^{b} y^{c} .
$$

Note that the sum in $I[g]$ extends over even powers in $\vec{\pi}$, while $\Gamma[g]$ contains the terms odd in $\pi$. Observe also that $I[g]$ and $\Gamma[g]$ are both real.

For the Euclidean WZNW action we choose the metric $\eta^{\mu \nu}=\operatorname{diag}(+,+)$, the Levi-Civitá symbol with $\varepsilon^{12}=1$ and define

$$
S_{W}^{ \pm}[g]=k(I[g] \pm i \Gamma[g]) .
$$

Here $k$ is an integer coupling constant such that the action is defined modulo an integer times $2 \pi i$ and $\exp S_{W}^{ \pm}$is unambiguous. Introducing complex coordinates,

$$
x^{ \pm}=\frac{\lambda}{\sqrt{2}}\left(x^{1} \pm i x^{2}\right), \quad \partial_{ \pm}=\frac{1}{\lambda \sqrt{2}}\left(\partial_{1} \mp i \partial_{2}\right)
$$


(so that $\partial_{\mu} \partial^{\mu}=2 \lambda^{2} \partial_{+} \partial_{-}$), where $\lambda$ is an arbitrary normalization factor to facilitate comparison with corresponding expressions in the literature円 the action satisfies the Polyakov-Wiegmann formula [7]

$$
S_{W}^{ \pm}[g h]=S_{W}^{ \pm}[g]+S_{W}^{ \pm}[h]+\frac{k \lambda^{2}}{4 \pi \chi} \int \mathrm{d}^{2} x \operatorname{Tr} \partial_{ \pm} h h^{-1} g^{-1} \partial_{\mp} g .
$$

Combining (2.3) and (2.4) gives

$S_{W}^{ \pm}[g]=\frac{k \lambda^{2}}{4 \pi} \int \mathrm{d}^{2} x\left\{-\frac{1}{2} \partial_{+} \vec{\pi} \cdot \partial_{-} \vec{\pi} \mp \frac{1}{6} \vec{\pi} \cdot\left(\partial_{+} \vec{\pi} \times \partial_{-} \vec{\pi}\right)+\frac{1}{24}\left(\vec{\pi} \times \partial_{+} \vec{\pi}\right) \cdot\left(\vec{\pi} \times \partial_{-} \vec{\pi}\right)+\mathcal{O}\left(\pi^{5}\right)\right\}$.

Let us define $A_{+}=\partial_{+} g g^{-1}=\left(A_{1}-i A_{2}\right) /(\lambda \sqrt{2})$. Under left multiplication of $g$ with an $x$-dependent element of the group near the identity, $A_{+}$transforms as

$$
\delta \vec{A}_{+}=\partial_{+} \vec{\eta}+\vec{\eta} \times \vec{A}_{+}
$$

where $\eta^{a} T_{a}=\delta g g^{-1}$. Using (2.7) shows at once that the action changes under this variation as follows

$$
\begin{aligned}
\delta S_{W}^{+}[g] & =-\frac{k \lambda^{2}}{4 \pi \chi} \int \mathrm{d}^{2} x \operatorname{Tr} \delta g g^{-1} \partial_{-}\left(\partial_{+} g g^{-1}\right) \\
& =\frac{k \lambda^{2}}{4 \pi} \int \mathrm{d}^{2} x \vec{\eta}(x) \cdot \partial_{-} \vec{A}_{+}(x)
\end{aligned}
$$

The first equality shows the well-known result that $A_{+}$is holomorphic by virtue of the field equations (applying the same procedure with respect to right multiplication yields the conjugate result that $g^{-1} \partial_{-} g$ is anti-holomorphic), while the second equality takes the form of an anomalous variation, as we discuss below. Corresponding results can be obtained for $S_{W}^{-}[g]$ and $A_{-}=\partial_{-} g g^{-1}$.

The WZNW action is related to the induced action $S_{i n d}^{ \pm}\left[A_{ \pm}\right]$obtained from coupling a chiral system, such as a chiral spinor, to a chiral gauge field. It thus depends on the gauge fields $A_{+}^{a}$ or $A_{-}^{a}$ in a form which is purely two-dimensional, though nonlocal. Because of the chiral anomaly the induced action satisfies an anomalous Ward identity; under gauge transformations $\delta \vec{A}_{ \pm}=\partial_{ \pm} \vec{\eta}+\vec{\eta} \times \vec{A}_{ \pm}$it will transform ast2

$$
\delta S_{i n d}^{ \pm}\left[A_{ \pm}\right]=\frac{k \lambda^{2}}{4 \pi} \int \mathrm{d}^{2} x \vec{\eta}(x) \cdot \partial_{\mp} \vec{A}_{ \pm}(x) .
$$

The right-hand side originates from the anomaly of the underlying chiral theory. This anomaly is the unique (local) solution of the Wess-Zumino consistency equations. As there is only one (gauge) field it is not possible to construct gauge-invariant

\footnotetext{
${ }^{1}$ Occasionally we will work in Minkowski space, where we use

$$
x^{ \pm}=\frac{\lambda}{\sqrt{2}}(x \pm t), \quad \partial_{ \pm}=\frac{1}{\lambda \sqrt{2}}\left(\partial_{x} \pm \partial_{t}\right), \quad S_{W}^{ \pm}[g]=k(I[g] \pm \Gamma[g]) .
$$

${ }^{2} \mathrm{As}$ is well known, this equation is equivalent to the statement that the two-dimensional vector potential $\left(A_{ \pm}, u_{\mp}\right)$, with $u_{\mp}=-\left(4 \pi / k \lambda^{2}\right) \delta S_{i n d}^{ \pm}\left[A_{ \pm}\right] / \delta A_{ \pm}$, is curvature-free.
} 
actions in terms of this field, so that the induced action is uniquely determined by the anomalous Ward identity.

The solution can be determined directly by considering an explicit definition of the induced action in terms of matter currents (we choose $\lambda=\sqrt{2}$ ),

$$
\exp \left(S_{\text {ind }}^{+}\left[A_{+}\right]\right)=\left\langle 0\left|\exp \left(-\frac{1}{\pi \chi} \int \mathrm{d}^{2} x \operatorname{Tr} J(x) A_{+}(x)\right)\right| 0\right\rangle .
$$

Here, the matter currents satisfy the operator-product expansion of a Kac-Moody algebra,

$$
J_{a}(x) J_{b}(y) \sim \frac{\frac{1}{2} k g_{a b}}{\left(x^{-}-y^{-}\right)^{2}}+\frac{f_{a b}^{c} J_{c}(y)}{x^{-}-y^{-}}+\cdots .
$$

Using that vacuum-expectation values of normal-ordered products (the regular terms in the operator-product expansion) or of a single current vanish, one verifies by exploiting the standard identity $\partial_{+}\left(x^{-}\right)^{-1}=\pi \delta^{2}(x)$, that the logarithm of (2.12) satisfies the anomaly equation (2.11). Furthermore, expanding (2.12) to all orders in the gauge field and making repeated use of the operator-product expansion (2.13), gives an explicit representation for the induced action [11],

$$
\begin{aligned}
S_{i n d}^{+}\left[A_{+}\right]=\frac{k \lambda^{2}}{4 \pi \chi} \sum_{n=0}^{\infty} \frac{1}{2+n} \operatorname{Tr} \int \mathrm{d}^{2} x A_{+}\left[\frac{1}{\partial_{+}} A_{+}, \cdots\left[\frac{1}{\partial_{+}} A_{+}, \frac{\partial_{-}}{\partial_{+}} A_{+}\right] \cdots\right]_{n \text { times }} \\
=-\frac{k \lambda^{2}}{4 \pi} \int \mathrm{d}^{2} x \vec{A}_{+} \cdot\left[\frac{1}{2} \frac{\partial_{-}}{\partial_{+}} \vec{A}_{+}+\frac{1}{3} \frac{1}{\partial_{+}}\left(\vec{A}_{+} \times \frac{\partial_{-}}{\partial_{+}} \vec{A}_{+}\right)\right. \\
\left.+\frac{1}{4} \frac{1}{\partial_{+}}\left(\vec{A}_{+} \times \frac{1}{\partial_{+}}\left(\vec{A}_{+} \times \frac{\partial_{-}}{\partial_{+}} \vec{A}_{+}\right)\right)+\cdots\right]
\end{aligned}
$$

and likewise for $S_{i n d}^{-}\left[A_{-}\right]$. This result follows also from Feynman diagram calculations of the type considered in later sections.

The correspondence with the WZNW actions follows from the fact that $S_{i n d}^{ \pm}\left[A_{ \pm}\right]$ with $A_{ \pm}=\partial_{ \pm} g g^{-1}$ are solutions to the same anomalous Ward identity (2.10) as the WZNW actions. Hence we conclude that

$$
S_{i n d}^{+}\left[A_{+}(g)\right]=S_{W}^{+}[g], \quad S_{i n d}^{-}\left[A_{-}(\bar{g})\right]=S_{W}^{-}[\bar{g}],
$$

with $A_{+}(g)=\partial_{+} g g^{-1}$ and $A_{-}(\bar{g})=\partial_{-} \bar{g} \bar{g}^{-1}$ and $g(x)$ and $\bar{g}(x)$ two independent group-valued fields.

Combining these results with (2.7) gives [7]

$$
\begin{aligned}
S_{i n d}^{+}\left[A_{+}\right]+S_{i n d}^{-}\left[A_{-}\right] & =S_{W}^{+}[g]+S_{W}^{-}[\bar{g}] \\
& =S_{W}^{+}\left[\bar{g}^{-1} g\right]+\frac{k \lambda^{2}}{4 \pi \chi} \int \mathrm{d}^{2} x \operatorname{Tr} \partial_{+} g g^{-1} \partial_{-} \bar{g} \bar{g}^{-1} \\
& =S_{W}^{+}\left[\bar{g}^{-1} g\right]-\frac{k \lambda^{2}}{4 \pi} \int \mathrm{d}^{2} x \vec{A}_{+} \cdot \vec{A}_{-},
\end{aligned}
$$

which shows the well-known result that the sum of the two chiral induced actions yields a covariant action up to a local term quadratic in $A$. This term is related to the ultraviolet subtraction one has to perform in order to preserve gauge invariance of the covariant (nonchiral) theory. 


\section{$3 \quad$ Quantization and the background field method}

In the background field formulation of the WZNW action $S_{W}^{+}[g]$ (see e.g. [3, 4]) one replaces the group element by the product of two group elements, say $g \rightarrow h g$, where $g$ is the background field and $h$ is expanded with respect to the real quantum fields $\pi^{a}$ according to $h=\exp \pi^{a} T_{a}$. The background field only appears through the current $J_{+}=\partial_{+} g g^{-1}$, as follows from application of (2.7),

$$
S_{W}^{+}[h g]-S_{W}^{+}[g]=S_{W}^{+}[h]+\frac{k \lambda^{2}}{4 \pi \chi} \int \mathrm{d}^{2} x \operatorname{Tr} J_{+} h^{-1} \partial_{-} h .
$$

Both sides of this equation are invariant under

$$
\begin{aligned}
g(x) & \rightarrow U\left(x^{+}\right) g(x) \\
h(x) & \rightarrow U\left(x^{+}\right) h(x) U^{-1}\left(x^{+}\right) \\
J_{+}(x) & \rightarrow \partial_{+} U\left(x^{+}\right) U^{-1}\left(x^{+}\right)+U\left(x^{+}\right) J_{+}(x) U^{-1}\left(x^{+}\right) .
\end{aligned}
$$

Using the expression for $h^{-1} \partial_{-} h$ in terms of the quantum fields $\pi^{a}$ and the equalities (2.3) we deduce the following form of the action,

$S_{W}^{+}[h g]-S_{W}^{+}[g]=\frac{k \lambda^{2}}{4 \pi} \int \mathrm{d}^{2} x\left\{-\vec{J}_{+} \cdot \partial_{-} \vec{\pi}-\sum_{n=2}^{\infty} \frac{1}{n !} \partial_{-} \vec{\pi} \cdot\left(\vec{\pi} \times\left(\vec{\pi} \times \cdots D_{+} \vec{\pi}\right)\right)\right\}$,

with

$$
D_{+} \vec{\pi}=\partial_{+} \vec{\pi}-\vec{J}_{+} \times \vec{\pi}
$$

A similar expression can be derived for $S_{W}^{-}[\bar{g}]$ using the background field splitting $\bar{g} \rightarrow \bar{g} h^{-1}$. One obtains then an action involving $J_{-}=\partial_{-} \bar{g} \bar{g}^{-1}$,

$$
\begin{aligned}
S_{W}^{-}\left[h^{-1} \bar{g}\right]-S_{W}^{-}[\bar{g}] & =S_{W}^{+}[h]-\frac{k \lambda^{2}}{4 \pi \chi} \int \mathrm{d}^{2} x \operatorname{Tr} J_{-} \partial_{+} h h^{-1} \\
& =\frac{k \lambda^{2}}{4 \pi} \int \mathrm{d}^{2} x\left\{\vec{J}_{-} \cdot \partial_{+} \vec{\pi}-\sum_{n=2}^{\infty} \frac{1}{n !} D_{-} \vec{\pi} \cdot\left(\vec{\pi} \times\left(\vec{\pi} \times \cdots \partial_{+} \vec{\pi}\right)\right)\right\},
\end{aligned}
$$

which is invariant under

$$
h(x) \rightarrow U\left(x^{-}\right) h(x) U^{-1}\left(x^{-}\right), \quad \bar{g}(x) \rightarrow U\left(x^{-}\right) \bar{g}(x) .
$$

Note that with the exception of the term linear in $\vec{\pi}$, which is separately invariant, (3.3) and (3.5) follow from covariantizing $S_{W}^{+}[h]$, making use of the equalities (2.3). Alternatively, one can deduce the explicit form of (2.3) from requiring that the terms proportional to $J_{ \pm}$must follow from the covariantization of some action.

However, we are interested in neither the former chiral action, nor the latter. Rather, we prefer a covariant action containing both $J_{+}$and $J_{-}$with a local gauge invariance, since this will simplify our quantum calculations considerably as we shall 
discuss later. It is not hard to find an action involving $g, h$ and $\bar{g}$ with a local gauge invariance, namely the gauged WZNW model [22] described by

$$
S[\vec{\pi}, \vec{J}]=S_{W}^{+}\left[\bar{g}^{-1} h g\right]-S_{W}^{+}\left[\bar{g}^{-1} g\right],
$$

which is manifestly invariant under

$$
\begin{aligned}
& h(x) \rightarrow U(x) h(x) U^{-1}(x), \\
& g(x) \rightarrow U(x) g(x), \\
& \bar{g}(x) \rightarrow U(x) \bar{g}(x) .
\end{aligned}
$$

The latter two tranformation laws induce the standard gauge transformations for the currents

$$
J_{ \pm}(x) \rightarrow \partial_{ \pm} U(x) U^{-1}(x)+U(x) J_{ \pm}(x) U^{-1}(x) .
$$

Moreover, for $\bar{g}=\mathbf{1}$ we recover (3.3) and for $g=\mathbf{1}$ we recover (3.5) (using $S_{W}^{-}[\bar{g}]=$ $\left.S_{W}^{+}\left[\bar{g}^{-1}\right]\right)$.

The covariant action can be evaluated by fully covariantizing the actions (3.3) and (3.5). The result is

$$
\begin{aligned}
S[\vec{\pi}, \vec{J}]= & S_{W}^{+}[h]-\frac{k \lambda^{2}}{4 \pi \chi} \int \mathrm{d}^{2} x \operatorname{Tr}\left[\partial_{+} h h^{-1} J_{-}-J_{+} h^{-1} \partial_{-} h+J_{+} h^{-1}\left[J_{-}, h\right]\right] \\
= & -\frac{k}{8 \pi} \int \mathrm{d}^{2} x i \varepsilon^{\mu \nu} \vec{\pi} \cdot\left(\partial_{\mu} \vec{J}_{\nu}-\partial_{\nu} \vec{J}_{\mu}-\vec{J}_{\mu} \times \vec{J}_{\nu}\right) \\
& +\frac{k}{8 \pi} \int \mathrm{d}^{2} x \sum_{n=1}^{\infty}\left\{-\frac{1}{(2 n) !} D_{\mu} \vec{\pi} \cdot\left(\vec{\pi} \times\left(\vec{\pi} \times \cdots D^{\mu} \vec{\pi}\right)\right)\right. \\
& \left.\quad+\frac{i}{(2 n+1) !} \varepsilon^{\mu \nu} D_{\mu} \vec{\pi} \cdot\left(\vec{\pi} \times\left(\vec{\pi} \times\left(\vec{\pi} \times \cdots D_{\nu} \vec{\pi}\right)\right)\right)\right\},
\end{aligned}
$$

where the first term under the summation sign contains $2 n$ and the second $2 n+1$ fields $\vec{\pi}$, and the covariant derivative is given by

$$
D_{\mu} \vec{\pi}=\partial_{\mu} \vec{\pi}-\vec{J}_{\mu} \times \vec{\pi}
$$

Note again that the term linear in $\vec{\pi}$ does not follow from covariantizing the action (2.3), but is separately gauge invariant.

The last form of the action forms the basis of our subsequent calculations. We write down the Lagrangians for the terms of second, third and fourth order in the quantum fields $\vec{\pi}$. Dropping an overall factor $k / 8 \pi$, these Lagrangians are

$$
\begin{aligned}
\mathcal{L}^{(2)}= & -\frac{1}{2}\left(\partial_{\mu} \pi^{a}\right)^{2}-f^{a b c} J_{\mu}^{a} \partial^{\mu} \pi^{b} \pi^{c}-\frac{1}{2} f^{a b e} f^{c d e} J_{\mu}^{a} J^{c \mu} \pi^{b} \pi^{d}, \\
\mathcal{L}^{(3)}= & i \varepsilon^{\mu \nu}\left\{-\frac{1}{6} f^{a b c} \pi^{a} \partial_{\mu} \pi^{b} \partial_{\nu} \pi^{c}-\frac{1}{3} f^{a b c} f^{c d e} J_{\mu}^{a} \pi^{b} \pi^{d} \partial_{\nu} \pi^{e}\right. \\
& \left.+\frac{1}{6} f^{a c f} f^{f e g} f^{b d g} J_{\mu}^{a} J_{\nu}^{b} \pi^{c} \pi^{d} \pi^{e}\right\}, \\
\mathcal{L}^{(4)}= & \frac{1}{24} f^{a b e} f^{c d e} \partial_{\mu} \pi^{a} \pi^{b} \partial^{\mu} \pi^{c} \pi^{d}-\frac{1}{12} f^{a b f} f^{c f g} f^{d g e} J_{\mu}^{e} \partial^{\mu} \pi^{a} \pi^{b} \pi^{c} \pi^{d} \\
& +\frac{1}{24} f^{e a g} f^{g b h} f^{h c i} f^{i d f} J_{\mu}^{e} J^{f \mu} \pi^{a} \pi^{b} \pi^{c} \pi^{d} .
\end{aligned}
$$


In section 7 we shall discuss the relation of the action (3.10) with other background actions used in the literature [3, 6,8$]$. Occasionally we will also consider the WZNW action away from the critical point,

$$
S_{W}^{+}[g]=k\left(\frac{2 \pi}{k g^{2}} I[g]+i \Gamma[g]\right),
$$

where $g$ is some coupling constant (not to be confused with the group-valued fields). For the background action (3.10) this implies that one replaces the factor $k / 8 \pi$ in front of the terms even in $\vec{\pi}$ by $1 /\left(4 g^{2}\right)$.

\section{The one-loop background-field effective action in the WZNW model}

In this section we compute the one-loop contribution to the two- and three-point functions in the covariant background-field formulation developed in the previous section. These may be viewed as the one-particle irreducible contributions to the current correlation functions of the gauged WZNW model. The precise relation of our results to the quantum effective action of the WZNW model will be discussed in section 7. The section is divided into four parts. In subsection 4.1 we compute the (one-particle irreducible) two-point function for the WZNW model, using conventional dimensional-regularization techniques. In subsection 4.2 we repeat the calculation using complex function techniques, as an introduction to subsection 4.3 where we compute the (one-particle irreducible) three-point function using such techniques; the calculation is much simpler when done in this manner. Finally in subsection 4.4 we consider the connected two-point current correlation function and verify that it remains unrenormalized at the critical point, in accord with the work reported in 8 .

Although individual diagram contributions are ultraviolet divergent by power counting, the gauge invariance of the covariant action ensures the ultraviolet finiteness of the results of subsections 4.1-3. However, we encounter genuine infrared divergences, which, as we mentioned in the introduction, must be removed. Whereas at the one-loop level this could be achieved with conventional (e.g. mass) regularization, at the two-loop level such procedures are very cumbersome, and we prefer to use infrared subtraction methods as described in [25]. These methods treat momentumspace infrared singularities from vanishing denominators by complete analogy with $x$-space ultraviolet singularities. Thus one has the notion of subdivergences, overlapping divergences, and a full technology similar to the conventional BPHZ procedure. Essentially, infrared singularities are removed by adding suitable counterterms, local in momentum space, i.e. momentum $\delta$-functions and derivatives thereof. We refer the reader to the literature, but will explain the appropriate procedure whenever we encounter the need for it. Some specific equations and the treatment of a two-loop integral with overlapping infrared divergences is presented in the appendix.

The result of this section is that the one-loop effective action (corresponding to the one-particle irreducible graphs) is again proportional to the WZNW action 
Figure 1: One-loop diagrams contributing to the two- and three-point functions.

with the level $k$ shifted to $k+\tilde{h}$, up to logarithmic terms associated with infrared divergences.

\subsection{The two-point function}

For this calculation, and in the future, we shall omit the factor $k / 8 \pi$ (a loop counting parameter essentially) and insert it back only at the end of the calculation. For the one-loop diagrams we only need the Lagrangian $\mathcal{L}^{(2)}$, given in (3.12), which coincides with the Lagrangian of scalar Yang-Mills theory.

The two-point function follows from the first two graphs in Fig. 1 and leads to the expression

$$
\Pi_{\mu \nu}^{a b}(p)=\tilde{h} g^{a b} \int \frac{\mathrm{d}^{2} k}{(2 \pi)^{2}}\left[\frac{k_{\mu} k_{\nu}-(p-k)_{\mu} k_{\nu}}{k^{2}(p-k)^{2}}-\frac{\delta_{\mu \nu}}{k^{2}}\right] .
$$

The individual terms are superficially ultraviolet logarithmically divergent but gauge invariance requires the two-point function to be transverse, thus ensuring the ultraviolet finiteness of the answer. They are also infrared divergent and these divergences must be subtracted out. We use dimensional regularization with

$$
d=2-2 \epsilon,
$$

and one-loop infrared counterterms for simple propagators which amount to the replacement

$$
\frac{1}{k^{2}} \rightarrow \frac{1}{k^{2}}+\frac{\pi}{\epsilon} \delta^{(2)}(k)
$$

The integrals can be done now by conventional dimensional regularization. In particular integrals over a single massless propagator may be set to zero. (They vanish because of cancellations between ultraviolet and infrared divergences, but since we wish to keep track of ultraviolet divergent terms, which are expected to cancel among themselves, this can be done only after including the infrared counterterms.)

We continue momentum integrals into $d$ dimensions by introducing an additional factor $\Gamma(1-\epsilon)(4 \pi)^{-\epsilon}$ for each loop. It is known that this procedure - the so-called G-scheme [26] - removes contributions proportional to $\ln 4 \pi$, to the Euler constant $\gamma_{E}$ and also, at higher loops, terms proportional to the Riemann function $\zeta(2)$, and 
corresponds to a form of modified minimal subtraction, but in our case applied to infrared rather than ultraviolet divergences. We use in particular

$$
\begin{aligned}
& \Gamma(1-\epsilon)(4 \pi)^{-\epsilon} \int \frac{\mathrm{d}^{d} k}{(2 \pi)^{d}} \frac{1}{\left[k^{2}\right]^{\alpha}\left[(k-p)^{2}\right]^{\beta}}= \\
& \frac{1}{4 \pi} \frac{\Gamma(1-\epsilon) \Gamma(\alpha+\beta-1+\epsilon) \Gamma(1-\epsilon-\alpha) \Gamma(1-\epsilon-\beta)}{\Gamma(\alpha) \Gamma(\beta) \Gamma(2-2 \epsilon-\alpha-\beta)} \frac{1}{\left[p^{2}\right]^{\alpha+\beta-1+\epsilon}} .
\end{aligned}
$$

Other useful integrals derived from this are listed in the appendix.

The evaluation of (4.1) is now straightforward. The answer is proportional to the transverse projection operator $\left(\delta_{\mu \nu}-p_{\mu} p_{\nu} / p^{2}\right)$, as required by gauge invariance,

$$
\Pi_{\mu \nu}^{a b}(p)=\frac{\tilde{h}}{4 \pi} g^{a b} \frac{[\Gamma(1-\epsilon)]^{3} \Gamma(1+\epsilon)}{\epsilon \Gamma(2-2 \epsilon)}\left[\frac{p^{2}}{\mu^{2}}\right]^{-\epsilon}\left(\delta_{\mu \nu}-\frac{p_{\mu} p_{\nu}}{p^{2}}\right),
$$

where $\mu$ is the mass parameter required by dimensional regularization. The pole at $\epsilon=0$ represents an infrared singularity and is removed by the infrared counterterms discussed above. It amounts to adding to this result the expression

$$
\Delta_{I R} \Pi_{\mu \nu}^{a b}(p)=\tilde{h} g^{a b} \int \frac{\mathrm{d}^{2} k}{(2 \pi)^{2}}\left[\frac{\pi}{\epsilon} \delta^{(2)}(p-k) \frac{k_{\mu} k_{\nu}}{k^{2}}-\frac{\pi}{\epsilon} \delta^{(2)}(k) \delta_{\mu \nu}\right] .
$$

After this subtraction we obtain

$$
\Pi_{\mu \nu}^{a b}(p)=\frac{\tilde{h}}{4 \pi} g^{a b}\left(2-\ln \frac{p^{2}}{\mu^{2}}\right)\left(\delta_{\mu \nu}-\frac{p_{\mu} p_{\nu}}{p^{2}}\right) .
$$

We could also have used a regulator mass in the above calculation. In that case the result is finite for finite regulator mass, so one can take the limit $d \rightarrow 2$. However, now the logarithm emerges in the limit that the regulator mass is taken to zero, and the result takes the same form with $\mu$ the regulator mass. The reason that this regularization leads to an identical result is that the theory is ultraviolet finite, so that the emergence of $\ln p^{2} / \mu^{2}$ is entirely an infrared effect.

Ignoring the $\ln p^{2} / \mu^{2}$ we find the following effective action to order $J^{2}$,

$$
S_{e f f}[J]=-\frac{\tilde{h}}{8 \pi} \int \mathrm{d}^{2} x g_{a b}\left(\partial_{\mu} J_{\nu}^{a}-\partial_{\nu} J_{\mu}^{a}\right) \frac{1}{\square}\left(\partial^{\mu} J^{\nu a}-\partial^{\nu} J^{\mu a}\right) .
$$

The same calculation for Dirac fermions rather than for scalars yields -2 times the above result, but this time without the $\ln p^{2} / \mu^{2}$ terms! This is due to the fact that the numerator of the first term in (4.1) acquires an extra term equal to $\frac{1}{2}\left(p^{2} \delta_{\mu \nu}-p_{\mu} p_{\nu}\right)$. The fermionic result is in accord with that of the abelian Schwinger model, where it is responsible for generating a gauge-field mass equal to $e^{2} / \pi$ (in that case the fermions do not transform according to the adjoint representation, so that $\tilde{h}$ is replaced by $e^{2}$ ). The absence of the $\ln p^{2} / \mu^{2}$ terms can be understood from the fact that the fermion fields are primary operators. For the scalars this is different; unlike the currents $J_{ \pm}$, the quantum fields $\vec{\pi}$ are not primary. Only after summing the logarithms to all orders of perturbation theory one may expect to find finite results in the limit $\mu \rightarrow 0$ (or, if one had not subtracted the infrared divergences 
and stayed in $d$ dimensions, in the limit $d \rightarrow 2$ ). Our approach is therefore to simply drop the $\ln p^{2} / \mu^{2}$ terms, assuming that their summation to all orders leads to a vanishing contribution (consider, for example, the power series expansion of $\ln \frac{p^{2}}{\mu^{2}}\left[1-\ln \frac{p^{2}}{\mu^{2}}\right]^{-2}$, which vanishes when $\left.\mu \rightarrow 0\right)$. In the case at hand, comparison with the fermionic effective action seems to justify this approach. Beyond one-loop order there are no fermionic diagrams, which seems in line with the result derived in section 6 , where we find that the entire two-loop correction for the WZNW theory is proportional to $\ln ^{2} p^{2} / \mu^{2}$.

One may ask whether the presence of the infrared divergences is not at variance with the so-called Elitzur conjecture [27, 28, 29]. This conjecture pertains to theories with Goldstone bosons, and asserts the infrared finiteness of Green's functions of operators that are invariant under the group that is spontaneously broken and thus responsible for the zero mass of the fields. An intuitive way to understand this conjecture is to observe that the zero modes responsible for the infrared divergences can be eliminated by imposing a "gauge condition" (such as fixing the values of the fields at a given point). For invariant operators the Green's functions are independent of this condition, so that they should be infrared finite without the need for additional conditions or subtractions [28].

In the context of our work, the relevant symmetry corresponds to left (or right) group multiplication of the quantum field $h$, which leaves the theory invariant provided we restrict ourselves to the currents $J_{+}\left(\right.$or $\left.J_{-}\right)$. Consequently, we may conclude that the infrared divergences must cancel when restricting ourselves to connected Green's functions associated with $J_{+}$( or $J_{-}$). This is indeed the case, as we know from [8] that these Green's functions are in fact not renormalized and given by their classical values. We shall discuss this for the two-point function in subsection 4.4. The origin of the infrared divergences in the effective action is thus related to the fact that the concept of one-particle irreducible diagrams refers to the quantum fields $\vec{\pi}$, which are not invariant under the relevant group, and thus not subject to the Elitzur conjecture.

Returning to the result of our calculation, we restrict ourselves to the $J_{+^{-}}$ dependent part of (4.8), which reads

$$
S_{e f f}\left[J_{+}\right]=-\frac{\tilde{h} \lambda^{2}}{8 \pi} \int \mathrm{d}^{2} x \vec{J}_{+} \cdot \frac{\partial_{-}}{\partial_{+}} \vec{J}_{+} .
$$

Comparing this one-loop result to the classical action $S_{W}^{+}$, or equivalently, to $S_{i n d}^{+}\left[J_{+}(g)\right]$, suggests that the level $k$ in the classical action is now renormalized to $k+\tilde{h}$, which is the result stated at the beginning of this section. This conclusion will be confirmed by the calculation of the three-point function in subsection 4.3. Another observation, which is useful later on, is that comparison of (4.9) to (2.14) shows that the action induced by a chiral fermion is equal to $-(2 \chi / k) S_{\text {ind }}^{ \pm}\left[A_{ \pm}\right]$, where $\chi$ is the index of the fermion representation. 


\subsection{The two-point function by analytic methods}

Here we concentrate directly on the computation of the $J_{+}$part of the effective action. The procedure we use has been known for almost 30 years [24]. We do the calculation in Minkowski space, in terms of light-cone variables. Starting with the expression in $(4.1)$ we extract the term proportional to $J_{+a} J_{+b}$ in $\Pi_{\mu \nu}^{a b} J_{a}^{\mu} J_{b}^{\nu}$ and evaluate the integral

$$
\begin{aligned}
\Pi_{--}^{a b}(p) & =\frac{\lambda^{2} \tilde{h}}{4} g^{a b} \int \frac{\mathrm{d} k_{+} \mathrm{d} k_{-}}{i(2 \pi)^{2}} \frac{\left(2 k_{-}-p_{-}\right) k_{-}}{\left[k_{+} k_{-}-i \eta\right]\left[\left(k_{+}-p_{+}\right)\left(k_{-}-p_{-}\right)-i \eta\right]} \\
& =\frac{\lambda^{2} \tilde{h}}{8 \pi} g^{a b} \int_{0}^{p_{-}} \mathrm{d} k_{-} \frac{2 k_{-}-p_{-}}{k_{-}-p_{-}}\left(\frac{i \eta}{k_{-}}-p_{+}-\frac{i \eta}{k_{-}-p_{-}}\right)^{-1} \\
& =-\frac{\lambda^{2} \tilde{h}}{4 \pi p_{+}} g^{a b} \int_{0}^{p_{-}} \mathrm{d} k_{-} \frac{k_{-}^{2}-\frac{1}{2} k_{-} p_{-}}{k_{-}^{2}-k_{-} p_{-}+i \eta p_{-} / p_{+}} .
\end{aligned}
$$

We have performed the $k_{+}$integration by closing the contour in the complex $k_{+}$ plane, obtaining a non-zero result only if the two poles of the integrand are in opposite half-planes - this leads to the restriction on the $k_{-}$integration range.

To perform the $k_{-}$integration we decompose the integrand using partial fractions into a sum of two simple pole terms and a constant. We obtain

$$
\Pi_{--}^{a b}(p)=-\frac{\lambda^{2} \tilde{h}}{4 \pi p_{+}} g^{a b}\left[p_{-}+\frac{p_{-}^{2}}{2 R} \ln \frac{p_{-}-R}{p_{-}+R}\right], \quad R=\sqrt{p_{-}^{2}-4 i \eta \frac{p_{-}}{p_{+}}} .
$$

The limit $\eta \rightarrow 0$ gives then

$$
\Pi_{--}^{a b}(p)=-\frac{\lambda^{2} \tilde{h}}{8 \pi} g^{a b} \frac{p_{-}}{p_{+}}\left(2-\ln \frac{p^{2}}{2 i \eta}\right) .
$$

where $\eta$ is now the infinitesimal parameter that appears in the covariant Feynman integral (which differs from the one used in (4.10) by a factor $2 \lambda^{2}$ ). This result matches the previous one obtained in the covariant approach, provided we identify the two logarithms. We note that dropping the logarithm corresponds to dropping, in the integrand, the pole factors found after partial fraction expansion. When we compute the three-point function, by similar techniques, we will immediately drop the corresponding sources of logarithmic contributions.

\subsection{The three-point function by analytic methods}

There are two diagrams contributing to the three-point function, shown in Fig. 1. As the diagram (d) turns out to vanish by symmetric integration, we are left with the diagram (c), which leads to

$$
\Gamma_{\mu \nu \rho}^{a b c}\left(p^{a}, p^{b}, p^{c}\right)=\frac{1}{2} i \tilde{h} f^{a b c} \int \frac{\mathrm{d}^{2} k}{(2 \pi)^{2}} \frac{\left(2 k-p^{a}\right)_{\mu}\left(2 k+p^{b}\right)_{\nu}\left(2 k-p^{a}+p^{b}\right)_{\rho}}{k^{2}\left(k-p^{a}\right)^{2}\left(k+p^{b}\right)^{2}}
$$


where $p^{a}, p^{b}$ and $p^{c}$ are the incoming momenta associated with the background currents $J_{\mu}^{a}, J_{\nu}^{b}$ and $J_{\rho}^{c}$. In passing we note that this vertex function satisfies the Ward identity associated with (3.9),

$$
p^{a \mu} \Gamma_{\mu \nu \rho}^{a b c}\left(p^{a}, p^{b}, p^{c}\right)=i\left(f^{a c d} \Pi_{\nu \rho}^{b d}\left(p^{b}\right)+f^{a b d} \Pi_{\nu \rho}^{d c}\left(p^{c}\right)\right) .
$$

After continuing to Minkowski space we evaluate the integral by contour integration again, concentrating on the contribution cubic in $J_{+}$. It reads

$$
\begin{aligned}
& \Gamma_{---}^{a b c}\left(p^{a}, p^{b}, p^{c}\right)=\frac{1}{16} i \lambda^{2} \tilde{h} f^{a b c} \\
& \times \int \frac{\mathrm{d} k_{+} \mathrm{d} k_{-}}{i(2 \pi)^{2}} \frac{\left(2 k_{-}-p_{-}^{a}\right)\left(2 k_{-}+p_{-}^{b}\right)\left(2 k_{-}-p_{-}^{a}+p_{-}^{b}\right)}{\left[k_{+} k_{-}-i \eta\right]\left[\left(k_{+}-p_{+}^{a}\right)\left(k_{-}-p_{-}^{a}\right)-i \eta\right]\left[\left(k_{+}+p_{+}^{b}\right)\left(k_{-}+p_{-}^{b}\right)-i \eta\right]} .
\end{aligned}
$$

After taking partial fractions the integral over $k_{+}$decomposes into two integrals of the type encountered in subsection 4.2. This leaves us with a finite-range integral over $k_{-}$and we find

$$
\Gamma_{---}^{a b c}\left(p^{a}, p^{b}, p^{c}\right)=\frac{i \lambda^{2} \tilde{h}}{32 \pi} f^{a b c}\left(\frac{I\left(p^{a}\right)}{p_{+}^{c} p_{+}^{a}}+\frac{I\left(-p^{b}\right)}{p_{+}^{c} p_{+}^{b}}\right),
$$

where

$$
I(P)=\int_{0}^{P_{-}} \mathrm{d} k_{-} \frac{\left(2 k_{-}-p_{-}^{a}\right)\left(2 k_{-}+p_{-}^{b}\right)\left(2 k_{-}-p_{-}^{a}+p_{-}^{b}\right)\left(k_{-}-P_{-}\right)}{\left[\left(k_{-}+p_{-}^{b}\right)\left(k_{-}-p_{-}^{a}\right)-i \eta p_{-}^{c} / p_{+}^{c}\right]\left[k_{-}^{2}-k_{-} P_{-}-i \eta P_{-} / P_{+}\right]} .
$$

The integrands are rational functions of $k_{-}$, which can be separated, as in the previous subsection, into pole terms and a constant part. The pole terms lead once again to logarithms, and we drop them without further evaluation. The constant part equals $I(P)=8 P_{-}$, so that we obtain the result

$$
\Gamma_{---}^{a b c}\left(p^{a}, p^{b}, p^{c}\right)=\frac{i \lambda^{2} \tilde{h}}{4 \pi} f^{a b c}\left(\frac{p_{-}^{a}}{p_{+}^{c} p_{+}^{a}}-\frac{p_{-}^{b}}{p_{+}^{c} p_{+}^{b}}\right),
$$

Again it is illuminating to compare the result to that of the corresponding fermion theory. In that case the expression (4.13) acquires many extra terms. However, most of them do not contribute to the result cubic in $J_{+}$. The ones that do can easily be evaluated and lead to the following modification to the numerator $N$ in (4.15),

$$
N \rightarrow N+2 k_{-}\left(p_{-}^{a} p_{-}^{b}+p_{-}^{b} p_{-}^{c}+p_{-}^{c} p_{-}^{a}\right)+p_{-}^{a} p_{-}^{b}\left(p_{-}^{a}-p_{-}^{b}\right),
$$

which changes the numerator in $(4.17)$ to $8\left(k_{-}-p_{-}^{a}\right)\left(k_{-}+p_{-}^{b}\right) k_{-}\left(k_{-}-P_{-}\right)$. In the limit $\eta \rightarrow 0$ this leads then exactly to the result quoted in (4.18), without any logarithmic correction. This result is in accord with the analysis given at the end of subsection 4.1.

Finally, the above result corresponds to the following cubic term in the effective action,

$$
S_{e f f}\left[J_{+}\right]=\frac{-\lambda^{2} \tilde{h}}{12 \pi} f_{a b c} \int \mathrm{d}^{2} x J_{+}^{a} \frac{1}{\partial_{+}}\left(J_{+}^{b} \frac{\partial_{-}}{\partial_{+}} J_{+}^{c}\right) .
$$

This result and (4.9) constitute precisely the first two terms of the induced action up to an overall factor $\tilde{h} / k$, which confirms the assertion that the level $k$ changes to $k+\tilde{h}$. 


\subsection{The one-loop current correlation function}

In [7, 3] it was proven that the generating functional of current correlation functions in the WZNW model is unrenormalized. This was confirmed at the level of lagrangian field theory in [8]. Formally this result follows from (2.7) under the assumption that the measure in the path integral is invariant under left multiplication with an arbitrary ( $x$-dependent) group element. The generating functional of current correlation functions $W\left[J_{\mp}\right]$ is defined by

$$
\exp W\left[J_{\mp}\right]=\int \mathcal{D} g \exp \left(S_{W}^{ \pm}[g]-\frac{k \lambda^{2}}{4 \pi \chi} \int \mathrm{d}^{2} x \operatorname{Tr} J_{\mp} \partial_{ \pm} g g^{-1}\right),
$$

where $\mathcal{D} g$ is the Haar measure $\operatorname{det}\left(e_{i}^{a}(\phi)\right) \mathrm{d} \phi^{i}$ with $\mathrm{d} g g^{-1}=e_{i}^{a}(\phi) T_{a} \mathrm{~d} \phi^{i}$. Following [7], we substitute $J_{\mp}=\partial_{\mp} h h^{-1}$ and use (2.7) to rewrite the exponent on the righthand side in terms of the difference of two WZNW actions. Because of the invariance of the measure the integral leads to an $h$-independent factor and we are left with (up to an irrelevant additive constant)

$$
W\left[J_{\mp}\right]=-S_{W}^{\mp}[h]=-S_{\text {ind }}^{\mp}\left[J_{\mp}\right] .
$$

Thus the connected Green's functions are not renormalized and in particular no level shift appears here.

This result was verified at the one-loop level for the two-point correlation function of the critical theory in appendix A of [8]. We have repeated the calculation, but also away from the critical point where we replace the factor $k / 8 \pi$ in front of the terms even in the fields $\vec{\pi}$ in the Lagrangian (3.10) by $1 /\left(4 g^{2}\right)$ (cf. (3.15)), and we outline it here.

In tree approximation there is a single diagram for the connected two-point current correlation function, where a quantum field is exchanged between two currents. At the one-loop level we have additional one-particle reducible diagrams. The sum of all one-particle reducible graphs factorizes as $\Gamma_{\mu}(p) \Delta_{\pi}\left(p^{2}\right) \Gamma_{\nu}(-p)$, where $\Delta_{\pi}\left(p^{2}\right)$ is the quantum-field propagator and $\Gamma_{\mu}(p)$ the one-particle irreducible vertex of a current and a single quantum field. Furthermore there are the one-particle irreducible diagrams we evaluated in subsection 4.1. Here and in the remainder of this section we supress group indices.

Using the same regularization as before (so that the Haar measure does not contribute to the loops) we determine first the one-loop propagator

$$
\Delta_{\pi}\left(p^{2}\right)=4 g^{2} \frac{Z_{\pi}\left(p^{2}\right)}{p^{2}},
$$

for the quantum field. Here $Z_{\pi}$ receives contributions from a self-energy and a seagull diagram (using the cubic and quartic quantum field vertices in (3.13-14)) and is given by

$$
Z_{\pi}^{-1}\left(p^{2}\right)=1+\frac{\tilde{h}}{k}\left(\frac{k g^{2}}{2 \pi}\right)\left[-\frac{1}{6 \epsilon}+\left(\frac{k g^{2}}{2 \pi}\right)^{2}\left[\frac{1}{2 \epsilon}+1-\frac{1}{2} \ln \frac{p^{2}}{\mu^{2}}\right]\right] .
$$


In obtaining this result we subtracted an infrared divergence, but we are still left with a $1 / \epsilon$ pole corresponding to an ultraviolet divergence.

From this, and a similar calculation of the quantum-field three-point function (assuming that $k$ is not renormalized) we also determine the relevant one-loop renormalizations (with minimal subtraction), which in our conventions take the form

$$
\begin{aligned}
(\vec{\pi} / g)_{\mathrm{B}} & =\left[1+\frac{1}{12 \epsilon} \frac{\tilde{h}}{k}\left(\frac{k g^{2}}{2 \pi}\right)\left[1-3\left(\frac{k g^{2}}{2 \pi}\right)^{2}\right]\right] \vec{\pi} / g \\
g_{\mathrm{B}}^{2} & =\left[1-\frac{1}{2 \epsilon} \frac{\tilde{h}}{k}\left(\frac{k g^{2}}{2 \pi}\right)\left[1-\left(\frac{k g^{2}}{2 \pi}\right)^{2}\right]\right] g^{2} .
\end{aligned}
$$

We independently computed the coupling constant renormalization using the background field method [3, 4], obtaining the same result. At the critical point $\left(k g^{2}=\right.$ $2 \pi$ ) there are of course no divergences associated with the coupling constant, but we emphasize that the quantum field itself still requires ultraviolet subtractions.

The one-particle irreducible current-field vertex can be written as

$$
\Gamma_{\mu}(p)=\frac{k}{4 \pi} F_{\pi}\left(p^{2}\right) \varepsilon_{\mu \nu} p^{\nu}
$$

where $p$ is the incoming momentum associated with the quantum field. At one loop, $F_{\pi}$ receives contributions from two diagrams and we find

$$
F_{\pi}\left(p^{2}\right)=1+\frac{\tilde{h}}{k}\left(\frac{k g^{2}}{2 \pi}\right)\left[\frac{1}{6 \epsilon}+1-\frac{1}{2} \ln \frac{p^{2}}{\mu^{2}}\right] .
$$

Again we have subtracted an infrared divergence and we are left with an ultraviolet divergence. In the combined contribution for the reducible diagrams the ultraviolet divergence is absorbed into the coupling-constant renormalization given in (4.25), and we obtain

$$
\begin{aligned}
\frac{k^{2} g_{\mathrm{B}}^{2}}{(2 \pi)^{2}} \Gamma_{\mu}(p) & \frac{Z_{\pi}\left(p^{2}\right)}{p^{2}} \Gamma_{\nu}(-p)=-\frac{1}{2 \pi}\left(\delta_{\mu \nu}-\frac{p_{\mu} p_{\nu}}{p^{2}}\right) \\
& \times\left\{k\left(\frac{k g^{2}}{2 \pi}\right)+\tilde{h}\left(\frac{k g^{2}}{2 \pi}\right)^{2}\left[2-\left(\frac{k g^{2}}{2 \pi}\right)^{2}\right]\left(1-\frac{1}{2} \ln \frac{p^{2}}{\mu^{2}}\right)\right\} .
\end{aligned}
$$

At the critical point the one-loop terms cancel against (4.7), so that one is left with the classical contribution, in accord with the calculation performed in $[8$. However, our results above show that we disagree with the assertion in [8] that also the "pion decay constant" is left unrenormalized (see eq. (5.14) in that work).

Away from the critical point the connected current correlation function is infrared but not ultraviolet divergent. The absence of ultraviolet divergences (after performing the coupling constant renormalization) is not ensured by the usual nonrenormalization theorems for conserved currents. In two dimensions these theorems are less powerful, at least in the presence of the epsilon tensor; while in the generic

\footnotetext{
${ }^{3}$ Since in [8] only the correlation function with two currents $J_{+}$is evaluated, the one-loop irreducible seagull diagram of Fig. 11 did not contribute.
} 
case the Green's function $\Gamma_{\mu}$ of a conserved current with a single field must vanish for a symmetry that is not spontaneously broken, this is not so in two dimensions, where $\Gamma_{\mu}$ can be proportional to $\varepsilon_{\mu \nu} p^{\nu}$. If (4.28) would have turned out to be infinite, a separate independent counterterm proportional to the term linear in $\vec{\pi}$ in (3.10) would have been necessary. The presence of infrared divergences is not in disagreement with Elitzur's conjecture, as the current operators are no longer invariant under left or right multiplication of the quantum field $h$.

\section{One-loop calculations in the induced action}

In this section we perform calculations which parallel those of the previous section, but in the framework of the induced, nonlocal action (2.14), rather than the WZNW action. We work with nonlocal kinetic and interaction terms, in lightcone/complex coordinates, and perform conventional momentum-space calculations using Polyakov's exponential regularization [19]. In this nonlocal field theory the diagrams are superficially linearly divergent, but are rendered finite by symmetric integration (Lorentz invariance). However, because of the linear divergences, the finite results are somewhat ambiguous and, in particular, shifts in loop momenta have to be handled with care. Nevertheless, by adopting a certain routing scheme, as discussed in [20] it has been possible to obtain answers consistent with those obtained by other methods. Here we determine the one-loop contribution to the two-and three-point functions. From these calculation we determine the two multiplicative renormalization factors $Z_{k}$ and $Z_{A}$.

We will choose $\lambda=\frac{1}{2} \sqrt{2}$, so that we can make direct use of the formulae for momentum integrals given in [20]. The Lagrangian then has an overall factor $k / 8 \pi$, which will be suppressed until the end of the calculation. Complex coordinates and momenta are defined according to (2.6). In this way $\partial_{ \pm}$will correspond to $i k_{ \pm}$in momentum space. The propagator equals (we suppress factors $(2 \pi)^{2}$ )

$$
\Delta^{a b}(k)=g^{a b} \frac{k_{+}}{k_{-}} .
$$

The nonlocal vertices in this theory are rather complicated. To reduce the number of contractions it is convenient to make use of the following formula for the first-order variation of the induced action

$$
\begin{aligned}
\delta S_{\text {ind }}^{+}\left[A_{+}\right]=-\frac{k \lambda^{2}}{4 \pi} \int \mathrm{d}^{2} x \delta \vec{A}_{+} \cdot\left[\frac{\partial_{-}}{\partial_{+}} \vec{A}_{+}\right. & +\frac{1}{\partial_{+}}\left(\vec{A}_{+} \times \frac{\partial_{-}}{\partial_{+}} \vec{A}_{+}\right) \\
& \left.+\frac{1}{\partial_{+}}\left(\vec{A}_{+} \times \frac{1}{\partial_{+}}\left(\vec{A}_{+} \times \frac{\partial_{-}}{\partial_{+}} \vec{A}_{+}\right)\right)+\cdots\right],
\end{aligned}
$$

which follows from the anomalous Ward identity (2.11) by iteration (but can also be evaluated explicitly). Replacing $\delta \vec{A}_{+}$by $\vec{B}_{+}$, we can use the above equation to generate vertices where $\vec{B}_{+}$can be directly identified with one particular external line, while the fields denoted by $\vec{A}_{+}$still have to be identified in all possible ways with other external or internal lines. In this way the number of contractions for an 
Figure 2: One-loop diagrams contributing to the two- and three-point functions for the induced theory.

$n$-point vertex is reduced by a factor $n$. Hence we introduce the following notation for the vertices

$$
\begin{aligned}
\mathcal{V}^{(3)} & =\left(\frac{1}{\partial_{+}} \vec{B}_{+}\right) \cdot\left(\vec{A}_{+} \times \frac{\partial_{-}}{\partial_{+}} \vec{A}_{+}\right) \\
\mathcal{V}^{(4)} & =\left(\frac{1}{\partial_{+}} \vec{B}_{+}\right) \cdot\left(\vec{A}_{+} \times \frac{1}{\partial_{+}}\left(\vec{A}_{+} \times \frac{\partial_{-}}{\partial_{+}} \vec{A}_{+}\right)\right) \\
\mathcal{V}^{(5)} & =\left(\frac{1}{\partial_{+}} \vec{B}_{+}\right) \cdot\left(\vec{A}_{+} \times \frac{1}{\partial_{+}}\left(\vec{A}_{+} \times \frac{1}{\partial_{+}}\left(\vec{A}_{+} \times \frac{\partial_{-}}{\partial_{+}} \vec{A}_{+}\right)\right)\right) .
\end{aligned}
$$

For instance, for the three-pont vertex with incoming momenta $p, q, r$ we identify the line with momentum $p$ and group index $a$ with $\vec{B}_{+}$. The other two lines are then contracted with the fields $\vec{A}_{+}$. This can be done in two different ways, and we find

$$
\Gamma^{a b c}(p, q, r)=-i f^{a b c} \frac{q_{+} r_{-}-q_{-} r_{+}}{p_{+} q_{+} r_{+}}
$$

where the vertex momenta are taken incoming and factors of $(2 \pi)^{2}$ are again suppressed.

For future use we record some integrals extracted from the Table in Appendix A of [20],

$$
\begin{aligned}
& \frac{1}{\pi} \int \mathrm{d}^{2} k \frac{k_{-}^{n}}{(p+k)_{+}}=\frac{(-1)^{n}}{n+1} p_{-}^{n+1}, \quad n \geq 0 \\
& \frac{1}{\pi} \int \mathrm{d}^{2} k \frac{1}{(p+k)_{+} k_{-}}=\int_{p^{2}}^{\infty} \frac{\mathrm{d} t}{t} e^{-\epsilon t}, \\
& \frac{1}{\pi} \int \mathrm{d}^{2} k \frac{k_{+}}{(p+k)_{+} k_{-}}=-p_{+} \int_{p^{2}}^{\infty} \frac{\mathrm{d} t}{t} e^{-\epsilon t} .
\end{aligned}
$$

They were computed by regularizing ultraviolet divergences with a factor $\exp \left(-\epsilon k^{2}\right)$. They were then evaluated by breaking the integration range, typically, into $k^{2}<p^{2}$ and $k^{2}>p^{2}$, expanding denominators such as $\left[(p+k)_{+}\right]^{-1}$ in power series in $k_{+} / p_{+}$ or $p_{+} / k_{+}$respectively, and using symmetric integration.

The two-point function receives one-loop contributions from the self-energy and seagull graphs (a) and (b) in Fig. 2. Using (5.3), where we identify the $\vec{B}_{+}$fields 
with the external lines we obtain for the former

$$
\Pi_{1}^{a b}(p)=\frac{\tilde{h} g^{a b}}{p_{+}^{2}} \int \frac{\mathrm{d}^{2} k}{(2 \pi)^{2}}\left[\left(\frac{k_{-}}{k_{+}}\right)^{2}-\frac{(k+p)_{-}}{(k+p)_{+}} \frac{k_{-}}{k_{+}}\right] \frac{k_{+}}{k_{-}} \frac{(k+p)_{+}}{(k+p)_{-}} .
$$

The latter is obtained from $\mathcal{V}^{(4)}$, where we identify one of the external lines with $\vec{B}_{+}$while the second external line is identified in three ways with one of the fields $\vec{A}_{+}$. One of the three contributions vanishes since $f^{a b c}$ is traceless, while the other terms yield

$$
\Pi_{2}^{a b}(p)=\frac{\tilde{h} g^{a b}}{p_{+}} \int \frac{\mathrm{d}^{2} k}{(2 \pi)^{2}} \frac{1}{(p+k)_{+}}\left[\frac{k_{-}}{k_{+}}-\frac{p_{-}}{p_{+}}\right] \frac{k_{+}}{k_{-}} .
$$

In Polyakov's exponential regularization we choose a convergence factor $\exp \left(-\epsilon q_{i}^{2}\right)$ once for each internal line of momentum $q_{i}$ and average over all choices. Thus, for the seagull we introduce $\exp \left(-\epsilon k^{2}\right)$ while for the self-energy we compute once with the regulator $\exp \left(-\epsilon k^{2}\right)$ and once with the regulator $\exp \left(-\epsilon(k+p)^{2}\right)$, and take onehalf the sum; but in fact they both give the same result. With these rules there is no ambiguity due to different routings. For a discussion of these issues see the last section of [20]. Using the integrals above we find for the self-energy graph the contribution

$$
-g^{a b} \frac{\tilde{h}}{4 \pi} \frac{p_{-}}{p_{+}}\left[1+\int_{p^{2}}^{\infty} \frac{\mathrm{d} t}{t} e^{-\epsilon t}\right] .
$$

For the seagull graph we find precisely the opposite result, so that the total one-loop correction to the two-point function vanishes.

For the three-point function there are three graphs to be computed: a triangle graph (c), a self-energy-like graph (d) and a seagull graph (e), shown in Fig. 2. Using (5.3) the triangle graph yields

$$
\begin{aligned}
\Gamma_{1}^{a b c}(p, q, r)=-\frac{1}{2} i \tilde{h} & f^{a b c} \int \frac{\mathrm{d}^{2} k}{(2 \pi)^{2}} \frac{k_{+}}{k_{-}} \frac{\ell_{+}}{\ell_{-}} \frac{h_{+}}{h_{-}} \\
& \times\left(\frac{h_{+} k_{-}-h_{-} k_{+}}{p_{+} h_{+} k_{+}}\right)\left(\frac{k_{+} \ell_{-}-k_{-} \ell_{+}}{q_{+} k_{+} \ell_{+}}\right)\left(\frac{\ell_{+} h_{-}-\ell_{-} h_{+}}{r_{+} \ell_{+} h_{+}}\right),
\end{aligned}
$$

with $\ell=k+q$ and $h=k-p$. The integrand equals

$$
\frac{1}{p_{+} q_{+} r_{+}}\left[\left(\frac{k_{+} \ell_{-}}{k_{-} \ell_{+}}-\frac{k_{-} \ell_{+}}{k_{+} \ell_{-}}\right)+\left(\frac{h_{+} k_{-}}{h_{-} k_{+}}-\frac{k_{+} h_{-}}{k_{-} h_{+}}\right)+\left(\frac{\ell_{+} h_{-}}{\ell_{-} h_{+}}-\frac{\ell_{-} h_{+}}{\ell_{+} h_{-}}\right)\right],
$$

and is clearly symmetric in $k, \ell, h$. Thus, the three exponential regularizations all give the same result. We regulate with $\exp \left(-\epsilon k^{2}\right)$.

We note that in the absence of the regulator each pair of terms would naively give zero, by shifting integration momenta. However, although the original expression for the diagram is convergent, each pair of terms is linearly divergent and naive shifts cannot be performed. With the regulator present, it is still the case that the first two pairs of terms gives zero (using symmetric integration and antisymmetry of the integrand under interchange of the + and - components). Hence, only the last two terms contribute. 
We evaluate the integrals by writing, e.g.

$$
\begin{aligned}
\frac{\ell_{+} h_{-}}{\ell_{-} h_{+}} & =\frac{(k-p+q+p)_{+}(k+q-p-q)_{-}}{(k+q)_{-}(k-p)_{+}} \\
& =1-\frac{r_{+}}{(k-p)_{+}}+\frac{r_{-}}{(k+q)_{-}}-\frac{r_{+} r_{-}}{(k+q)_{-}(k-p)_{+}}
\end{aligned}
$$

The last term is only logarithmically divergent, so a shift of loop momentum is permissible, after which the integrals given in (5.7) can be used to evaluate the complete contribution. We find for the triangle graph

$$
\Gamma_{1}^{a b c}(p, q, r)=-\frac{i \tilde{h}}{4 \pi} f^{a b c} \frac{q_{+} r_{-}-q_{-} r_{+}}{p_{+} q_{+} r_{+}} .
$$

We consider next the self-energy-like graph (d) with a three-point and a fourpoint vertex. Using (5.3) (assigning index $a$ to the field $B$ ) and (5.4) (assigning index $b$ to $B$ ) we obtain

$$
\begin{aligned}
\Gamma_{2}^{a b c}(p, q, r)= & \frac{i \tilde{h}}{p_{+} q_{+}} f^{a b c} \int \frac{\mathrm{d}^{2} k}{(2 \pi)^{2}}\left[\frac{(p-k)_{+}}{(p-k)_{-}}-\frac{k_{+}}{k_{-}}\right] \\
\times & \left.\times \frac{k_{-}}{p_{+} k_{+}}+\frac{1}{2} \frac{k_{-}}{(r+k)_{+} k_{+}}-\frac{1}{2} \frac{r_{-}}{(r+k)_{+} r_{+}}\right] \\
& + \text {cyclic }\{p, q, r\} .
\end{aligned}
$$

The integrals are again straightforward to evaluate by tricks similar to the one used for the triangle graph and we find for the self-energy-like correction

$$
\begin{aligned}
\Gamma_{2}^{a b c}(p, q, r)= & \frac{i \tilde{h}}{8 \pi p_{+} q_{+} r_{+}} f^{a b c}\left[-6\left(q_{+} r_{-}-q_{-} r_{+}\right)+p_{-}(q-r)_{+} \int_{p^{2}}^{\infty} \frac{\mathrm{d} t}{t} e^{-\epsilon t}\right. \\
+q_{-}(r-p)_{+} & \left.\int_{q^{2}}^{\infty} \frac{\mathrm{d} t}{t} e^{-\epsilon t}+r_{-}(p-q)_{+} \int_{r^{2}}^{\infty} \frac{\mathrm{d} t}{t} e^{-\epsilon t}\right]
\end{aligned}
$$

Finally we consider the seagull graph (e) obtained from $\mathcal{V}^{(5)}$. There are five different ways to contract two lines into a closed loop (the sixth possibility vanishes as $f^{a b c}$ is traceless). For each of them there are again two ways to attach the remaining two external lines. Altogether we thus obtain

$$
\begin{aligned}
\Gamma_{3}^{a b c}(p, q, r)=\frac{i \tilde{h}}{p_{+}} f^{a b c} \int \frac{\mathrm{d}^{2} k}{(2 \pi)^{2}} \frac{k_{+}}{k_{-}} & \frac{1}{(k-p)_{+}} \frac{1}{p_{+}} \frac{r_{-}}{r_{+}}-\frac{1}{2} \frac{1}{(k-p)_{+}} \frac{1}{(r+k)_{+}} \frac{r_{-}}{r_{+}} \\
& +\frac{1}{2} \frac{1}{(k-p)_{+}} \frac{1}{(r+k)_{+}} \frac{k_{-}}{k_{+}} \\
& \left.-\frac{1}{r_{+}} \frac{1}{(k+r)_{+}} \frac{r_{-}}{r_{+}}+\frac{1}{r_{+}} \frac{1}{(k+r)_{+}} \frac{k_{-}}{k_{+}}\right] \\
-\{q \leftrightarrow r\} . &
\end{aligned}
$$

We handle the integrals as above, using partial fractions and momentum shifts for terms which are only logarithmically divergent. We obtain for the seagull correction 
to the three-point vertex

$$
\begin{array}{r}
\Gamma_{3}^{a b c}(p, q, r)=\frac{i \tilde{h}}{8 \pi p_{+} q_{+} r_{+}} f^{a b c}\left[3\left(q_{+} r_{-}-q_{-} r_{+}\right)-p_{-}(q-r)_{+} \int_{p^{2}}^{\infty} \frac{\mathrm{d} t}{t} e^{-\epsilon t}\right. \\
\left.-q_{-}(r-p)_{+} \int_{q^{2}}^{\infty} \frac{\mathrm{d} t}{t} e^{-\epsilon t}-r_{-}(p-q)_{+} \int_{r^{2}}^{\infty} \frac{\mathrm{d} t}{t} e^{-\epsilon t}\right] .
\end{array}
$$

The ultraviolet divergent integrals in the self-energy-like and seagull contributions cancel and we obtain a one-loop result proportional to the classical three-point vertex in (5.6). Adding the two (taking into account the factor $-k / 8 \pi$ ) gives

$$
\Gamma^{a b c}(p, q, r)=i \frac{k+\tilde{h}}{8 \pi} f^{a b c} \frac{q_{+} r_{-}-q_{-} r_{+}}{p_{+} q_{+} r_{+}} .
$$

Together with the absence of corrections to the two-point function this implies renormalization factors such that

$$
Z_{A}^{2} Z_{k}=1 . \quad Z_{A}^{3} Z_{k}=1+\tilde{h} / k
$$

so that

$$
Z_{A}=1+\tilde{h} / k, \quad Z_{k}=1-2 \tilde{h} / k .
$$

We will discuss this result in the concluding section of the paper.

\section{The two-loop two-point function in the WZNW model}

In this section we calculate the two-point function in the covariant background field action (3.9) for the WZNW model at two-loops. The ultraviolet divergent part of the WZWN model off the critical point has been computed by Bos [4 and can also be obtained from calculations of the $\beta$-function for nonlinear sigma models with torsion [5]. As discussed in these references, the correct treatment of these models requires that the $\varepsilon^{\mu \nu}$ tensor be defined as a two-dimensional object. It was shown by Bos [4] that, in addition to the expected one-loop divergences, there are also $1 / \epsilon$ poles whose cancellation requires counterterms proportional to the "evanescent" operator $\hat{\hat{\delta}}^{\mu \nu} \partial_{\mu} \vec{\pi} \cdot \partial_{\nu} \vec{\pi}$, where $\hat{\hat{\delta}}^{\mu \nu}$ is the Kronecker symbol for indices corresponding to the $d-2$ extra dimensions. On the other hand, if the epsilon tensor is treated as a $d$-dimensional object such evanescent counterterms are absent.

The calculations in subsections 6.1 and 6.2 are done without removing any ultraviolet subdivergences. Although there are the usual (i.e. non-evanescent) one-loop divergences associated with the quantum fields (even at the critical point), the corresponding counterterm insertions cancel among themselves, something that is a special feature of the background field method in this order. Perhaps somewhat surprisingly the combined result of the two-loop graphs that we calculate, is ultraviolet finite for both two- and $d$-dimensional epsilon tensors, even off the critical 
point (provided we restrict the indices and the momentum of the external currents to two dimensions). Actually, the leading $1 / \epsilon^{2}$ divergence cancels by itself, for any antisymmetric tensor $\varepsilon^{\mu \nu}$, something that can be understood from power-counting arguments combined with gauge invariance. At this stage the value of the finite result will depend on the particular prescription one adopts for the epsilon tensor. However, once the effect of the evanescent subdivergences is taken into account, the results become identical.

Our diagrams suffer also from infrared divergences. These must be subtracted out, following the procedure outlined earlier and this gives rise to logarithmic corrections, as before. There are some new features at the two-loop level and we will discuss them at the appropriate place. Just as in the one-loop case, the final answer for the two-point function must be transverse, as a result of gauge invariance. As the reader will appreciate, this provides an important check on our calculations.

The two-loop graphs contributing to the two-point function can be divided into two sets, which are separately gauge invariant as they involve vertices whose coupling constants are not related by the requirement of gauge invariance. The first set contains vertices obtained from $\mathcal{L}^{(2)}$ and $\mathcal{L}^{(4)}$ (cf. (3.12) and (3.14)), which do not involve the epsilon tensor. The corresponding diagrams are shown in Fig. 3 and their evaluation is discussed in subsection 6.1. Their combined result is ultraviolet finite. Away from the critical point these diagrams acquire a factor $k g^{2} / 2 \pi$.

The second class of two-loop diagrams involves two vertices contained in $\mathcal{L}^{(3)}$ (cf. (3.13)) and vertices from $\mathcal{L}^{(2)}$, and is thus proportional to two epsilon tensors. These diagrams are shown in Fig. 4. We have succeeded in calculating these diagrams for an arbitrary antisymmetric tensor $\varepsilon_{\mu \nu}$, so that at this stage we can postpone the subtleties with epsilon tensors in dimensional regularization. The final answer of these graphs exhibits four different structures that are separately gauge invariant for arbitrary epsilon tensor. As mentioned above the leading $1 / \epsilon^{2}$ divergence cancels in the final result, while the remaining $1 / \epsilon$ divergences cancel when one restricts the components and the momentum of the external currents to two dimensions. However, the treatment of epsilon tensors can affect the value of the final, finite, result. We deal with this in subsection 6.3 , where we also treat the issue of evanescent counterterms. Away from the critical point the second class of diagrams acquires a factor $\left(k g^{2} / 2 \pi\right)^{3}$.

In what follows it is convenient to uniformly extract a factor $(2 \pi)^{-4} \tilde{h}^{2} g^{a b}$. Furthermore we have a common factor $8 \pi / k$, originating from the prefactor of the background field action, so that

$$
\Pi_{\mu \nu}^{a b}(p)=\frac{1}{2 \pi^{3}} \frac{\tilde{h}^{2}}{k} g^{a b} \Pi_{\mu \nu}(p) .
$$

The final two-loop result obtained in this section then reads

$$
\Pi_{\mu \nu}(p)=\frac{1}{12} \pi^{2} \ln ^{2} \frac{p^{2}}{\mu^{2}}\left(\delta_{\mu \nu}-\frac{p_{\mu} p_{\nu}}{p^{2}}\right),
$$

at the critical point where $k g^{2}=2 \pi$. 
Figure 3: Two-loop diagrams without $\varepsilon$ tensors contributing to the two-point function. The second diagram (b) must be combined with its mirror diagram. There are two diagrams with seagull insertions collectively denoted by (c).

\subsection{Diagrams without $\varepsilon$ tensors}

There are five graphs that contribute here, shown in Fig. 3, which we group into four different expressions,

$$
\begin{aligned}
\Pi_{\mu \nu}^{(a)}(p) & =\frac{5}{24} \delta_{\mu \nu}\left[\int \frac{\mathrm{d}^{2} q}{q^{2}}\right]^{2}, \\
\Pi_{\mu \nu}^{(b)}(p) & =-\frac{5}{6} \int \frac{\mathrm{d}^{2} q}{q^{2}} \int \mathrm{d}^{2} k \frac{k_{\mu} k_{\nu}}{\left(k+\frac{1}{2} p\right)^{2}\left(k-\frac{1}{2} p\right)^{2}}, \\
\Pi_{\mu \nu}^{(c)}(p) & =\frac{1}{12} \int \frac{\mathrm{d}^{2} q}{q^{4}}\left[\frac{(2 q-p)_{\mu}(2 q-p)_{\nu}}{(q-p)^{2}}-\delta_{\mu \nu}\right] \int \mathrm{d}^{2} k \frac{k^{2}+q^{2}}{k^{2}}, \\
\Pi_{\mu \nu}^{(d)}(p) & =\frac{1}{2} \int \mathrm{d}^{2} q \frac{q_{\mu} q^{\rho}}{\left(q+\frac{1}{2} p\right)^{2}\left(q-\frac{1}{2} p\right)^{2}} \int \mathrm{d}^{2} k \frac{k_{\rho} k_{\nu}}{\left(k+\frac{1}{2} p\right)^{2}\left(k-\frac{1}{2} p\right)^{2}} .
\end{aligned}
$$

The answers follow directly from straightforward application of the integral (4.4) and the integrals given in the appendix. Aside from the use of infrared subtractions, this is a conventional calculation. For instance, in the first contribution, we add to the propagator the simple counterterm given by (4.3). It alone contributes, since now the integration of $q^{-2}$ gives zero. Similar infrared subtractions are performed in all the other contributions. Note that in the third contribution the first term in the $k$ integral gives zero in dimensional regularization, so that the preceding $q$ integral only has an ordinary infrared divergence associated with an integral over $q^{-2}$.

For the convenience of the reader, we list the answers for each of the graphs,

$$
\Pi_{\mu \nu}^{(a)}(p)=\frac{5 \pi^{2}}{24 \epsilon^{2}} \delta_{\mu \nu},
$$


Figure 4: Two-loop diagrams involving two $\varepsilon$ tensors contributing to the two-point function. The diagram (a) must be combined with its mirror diagram. The last diagram (f) vanishes.

$$
\begin{aligned}
\Pi_{\mu \nu}^{(b)}(p) & =-\frac{5 \pi^{2}}{12 \epsilon^{2}}\left[\frac{\Gamma(1+\epsilon)[\Gamma(1-\epsilon)]^{3}}{\Gamma(2-2 \epsilon)}\left(\delta_{\mu \nu}-\frac{p_{\mu} p_{\nu}}{p^{2}}\right) \frac{1}{\left[p^{2}\right]^{\epsilon}}+\frac{p_{\mu} p_{\nu}}{p^{2}}\right], \\
\Pi_{\mu \nu}^{(c)}(p) & =\frac{\pi^{2}}{6 \epsilon^{2}}\left[\frac{\Gamma(1+\epsilon)[\Gamma(1-\epsilon)]^{3}}{\Gamma(2-2 \epsilon)}\left(\delta_{\mu \nu}-\frac{p_{\mu} p_{\nu}}{p^{2}}\right) \frac{1}{\left[p^{2}\right]^{\epsilon}}-\frac{1}{2} \delta_{\mu \nu}+\frac{p_{\mu} p_{\nu}}{p^{2}}\right], \\
\Pi_{\mu \nu}^{(d)}(p) & =\frac{\pi^{2}}{8 \epsilon^{2}}\left[\left[\frac{\Gamma(1+\epsilon)[\Gamma(1-\epsilon)]^{3}}{\Gamma(2-2 \epsilon)}\right]^{2}\left(\delta_{\mu \nu}-\frac{p_{\mu} p_{\nu}}{p^{2}}\right) \frac{1}{\left[p^{2}\right]^{2 \epsilon}}+\frac{p_{\mu} p_{\nu}}{p^{2}}\right] .
\end{aligned}
$$

All $1 / \epsilon$ poles now characterize ultraviolet divergences. The total result reads

$$
\Pi_{\mu \nu}(p)=\frac{\pi^{2}}{8 \epsilon^{2}}\left[1-\frac{\Gamma(1+\epsilon)[\Gamma(1-\epsilon)]^{3}}{\Gamma(2-2 \epsilon)}\left[\frac{\mu^{2}}{p^{2}}\right]^{\epsilon}\right]^{2}\left(\delta_{\mu \nu}-\frac{p_{\mu} p_{\nu}}{p^{2}}\right) .
$$

As mentioned at the beginning of this section, the answer is transverse, which is a useful check on the correctness of our result. Moreover the answer is finite. Note that the subtraction of infrared divergences was crucial for obtaining this result. The above result (6.5) represents the full two-loop contribution to the current twopoint function in a nonlinear sigma model corresponding to the first term $I[g]$ of the WZNW Lagrangian. Expanding in powers of $\epsilon$ yields

$$
\Pi_{\mu \nu}(p)=\frac{\pi^{2}}{8}\left[2-\ln \frac{p^{2}}{\mu^{2}}\right]^{2}\left(\delta_{\mu \nu}-\frac{p_{\mu} p_{\nu}}{p^{2}}\right) .
$$

\subsection{Diagrams with $\varepsilon$ tensors}

We list first the expressions corresponding to the diagrams in Fig. 田,

$$
\Pi_{\mu \nu}^{(a)}(p)=\frac{1}{2} \varepsilon^{\mu \rho} \varepsilon^{\lambda \sigma} \int \mathrm{d}^{2} q \mathrm{~d}^{2} k \frac{(p-2 k)_{\nu} q_{\rho} q_{\lambda} k_{\sigma}}{q^{2}(k-q)^{2} k^{2}(p-k)^{2}}+(\mu \leftrightarrow \nu),
$$




$$
\begin{aligned}
\Pi_{\mu \nu}^{(b)}(p) & =\frac{1}{6} \varepsilon^{\mu \rho} \varepsilon^{\nu \sigma} \int \mathrm{d}^{2} q \mathrm{~d}^{2} k \frac{q_{\rho}(k-2 q)_{\sigma}}{q^{2}(k-q)^{2}(k-p)^{2}}, \\
\Pi_{\mu \nu}^{(c)}(p) & =-\frac{1}{2} \varepsilon^{\rho \sigma} \varepsilon^{\tau \lambda} \int \mathrm{d}^{2} q \mathrm{~d}^{2} k \frac{q_{\rho} q_{\tau} k_{\sigma} k_{\lambda}(2 k-p)_{\mu}(2 k-p)_{\nu}}{k^{4} q^{2}(k-q)^{2}(p-k)^{2}} \\
\Pi_{\mu \nu}^{(d)}(p) & =-\frac{1}{2} \varepsilon^{\rho \sigma} \varepsilon^{\tau \lambda} \int \mathrm{d}^{2} q \mathrm{~d}^{2} k \frac{q_{\mu} q_{\rho}(p-q)_{\tau} k_{\sigma}(2 k-p)_{\nu}(p-k)_{\lambda}}{q^{2} k^{2}(p-q)^{2}(p-k)^{2}(k-q)^{2}} \\
\Pi_{\mu \nu}^{(e)}(p) & =\frac{1}{2} \delta_{\mu \nu} \varepsilon^{\rho \sigma} \varepsilon^{\tau \lambda} \int \mathrm{d}^{2} q \mathrm{~d}^{2} k \frac{k_{\rho} q_{\sigma} k_{\tau} q_{\lambda}}{q^{4} k^{2}(k-q)^{2}} .
\end{aligned}
$$

We have not listed the contribution of the diagram (f), because it vanishes for symmetry reasons. The answers for the diagrams (a), (b), (c) and (e) can be obtained straightforwardly by means of the integrals listed in the appendix, for arbitrary antisymmetric $\varepsilon$ tensors. The evaluation of the diagram (d), on the other hand, is nontrivial for arbitrary $\varepsilon$ tensors. Therefore we first concentrate on the contributions from the other diagrams, which yield

$$
\begin{aligned}
& \Pi_{\mu \nu}^{(a)}(p)=-\frac{\pi^{2}}{4 \epsilon^{2}} \varepsilon_{\mu \rho} \varepsilon^{\rho \sigma}\left[\frac{\tilde{\Gamma}_{1}(\epsilon)}{1-2 \epsilon} \frac{p_{\nu} p_{\sigma}}{\left[p^{2}\right]^{1+\epsilon}}\right. \\
& \left.+\frac{\tilde{\Gamma}_{2}(\epsilon)}{(1-2 \epsilon)(1-3 \epsilon)}\left(\frac{1}{2} \delta_{\nu \sigma}-(1-\epsilon) \frac{p_{\nu} p_{\sigma}}{p^{2}}\right) \frac{1}{\left[p^{2}\right]^{2 \epsilon}}\right] \\
& +(\mu \leftrightarrow \nu) \\
& \Pi_{\mu \nu}^{(b)}(p)=-\frac{\pi^{2}}{6 \epsilon^{2}} \varepsilon_{\mu \rho} \varepsilon_{\nu \sigma}\left[\frac{p^{\rho} p^{\sigma}}{p^{2}}+\frac{\tilde{\Gamma}_{1}(\epsilon)}{1-2 \epsilon}\left(\frac{3}{2} \delta^{\rho \sigma}-(2-\epsilon) \frac{p^{\rho} p^{\sigma}}{p^{2}}\right) \frac{1}{\left[p^{2}\right] \epsilon}\right. \\
& \left.+\frac{\tilde{\Gamma}_{2}(\epsilon)}{1-3 \epsilon}\left(-\frac{3}{4} \delta^{\rho \sigma}+\frac{p^{\rho} p^{\sigma}}{p^{2}}\right) \frac{1}{\left[p^{2}\right]^{2 \epsilon}}\right] \\
& \Pi_{\mu \nu}^{(c)}(p)=-\frac{\pi^{2}}{4 \epsilon^{2}}\left[\frac{\tilde{\Gamma}_{1}(\epsilon)}{1-2 \epsilon} \frac{p_{\mu} p_{\nu}}{p^{2}}\left(\frac{\left(\varepsilon^{\rho \sigma} p_{\sigma}\right)^{2}}{\left[p^{2}\right]^{1+\epsilon}}+\frac{\varepsilon_{\rho \sigma} \varepsilon^{\rho \sigma}}{4(1-\epsilon)}\right)\right. \\
& +\frac{\tilde{\Gamma}_{2}(\epsilon)}{(1+\epsilon)(1-2 \epsilon)(2-3 \epsilon)(1-3 \epsilon)} \frac{1}{\left[p^{2}\right]^{2 \epsilon}} \\
& \times\left((1-\epsilon) \varepsilon_{\mu \rho} \varepsilon_{\nu}{ }^{\rho}+4 \epsilon^{2} \frac{p_{(\mu} \varepsilon_{\nu) \rho} \varepsilon^{\rho \sigma} p_{\sigma}}{p^{2}}\right. \\
& +\frac{1}{4} \varepsilon^{\rho \sigma} \varepsilon_{\rho \sigma}\left(2(1-\epsilon) \delta_{\mu \nu}-\left(2-\epsilon+\epsilon^{2}\right) \frac{p_{\mu} p_{\nu}}{p^{2}}\right) \\
& \left.\left.+\frac{\left(\varepsilon^{\rho \sigma} p_{\sigma}\right)^{2}}{p^{2}}\left(2 \epsilon(1-2 \epsilon) \delta_{\mu \nu}-\left(2-\epsilon-9 \epsilon^{2}+2 \epsilon^{3}\right) \frac{p_{\mu} p_{\nu}}{p^{2}}\right)\right)\right], \\
& \Pi_{\mu \nu}^{(e)}(p)=\frac{\pi^{2}}{16 \epsilon^{2}} \delta_{\mu \nu} \varepsilon_{\rho \sigma} \varepsilon^{\rho \sigma} \frac{\tilde{\Gamma}_{1}(\epsilon)}{(1-\epsilon)(1-2 \epsilon)} .
\end{aligned}
$$

where we used the following definitions for the characteristic ratios of $\Gamma$ functions,

$$
\begin{aligned}
& \tilde{\Gamma}_{1}(\epsilon)=\frac{\Gamma(1+\epsilon)[\Gamma(1-\epsilon)]^{3}}{\Gamma(1-2 \epsilon)}=1-2 \zeta(3) \epsilon^{3}+\mathcal{O}\left(\epsilon^{4}\right), \\
& \tilde{\Gamma}_{2}(\epsilon)=\frac{\Gamma(1+2 \epsilon)[\Gamma(1-\epsilon)]^{5}}{\Gamma(1-3 \epsilon)}=1-10 \zeta(3) \epsilon^{3}+\mathcal{O}\left(\epsilon^{4}\right) .
\end{aligned}
$$


For future use we also recorded their expansion in $\epsilon$, which follows from (A.5) given in the appendix.

These diagrams have infrared divergences which were subtracted in the above expressions. The diagram (b) has overlapping infrared divergences, whose subtraction require special care. To illustrate the subtraction of two-loop infrared divergences, we present a more detailed treatment in the appendix.

Now we turn to the diagram $(\mathrm{d})$, which is more cumbersome because each subintegral involves at least three propagators. To evaluate this diagram we first bring it into a form that does not suffer from this complication. This is done by means of the following integration-by-parts procedure [26]. First one observes that

$$
\begin{aligned}
& (q-k)^{\kappa} \frac{\partial}{\partial q^{\kappa}}\left[\frac{\varepsilon^{\rho \sigma} \varepsilon^{\tau \lambda} q_{\rho} k_{\sigma}(p-q)_{\tau}(p-k)_{\lambda}}{q^{2} k^{2}(p-q)^{2}(p-k)^{2}(k-q)^{2}}\right] \\
& \quad=\frac{\varepsilon^{\rho \sigma} \varepsilon^{\tau \lambda} q_{\rho} k_{\sigma}(p-q)_{\tau}(p-k)_{\lambda}}{q^{2} k^{2}(p-q)^{2}(p-k)^{2}(k-q)^{2}}\left[-2+\frac{k^{2}}{q^{2}}-\frac{(q-k)^{2}}{q^{2}}+\frac{(p-k)^{2}}{(q-p)^{2}}-\frac{(q-k)^{2}}{(q-p)^{2}}\right] .
\end{aligned}
$$

Using this result one can perform a partial integration in the integral over $q$. After a reparametrization of loop momenta, one obtains the following expression for the diagram (d),

$$
\begin{aligned}
\Pi_{\mu \nu}^{(d)}(p)= & \frac{\varepsilon^{\rho \sigma} \varepsilon^{\tau \lambda}}{4 \epsilon(1-2 \epsilon)} \int \mathrm{d}^{2} q \mathrm{~d}^{2} k\left((2 k-p)_{\mu}+2 \epsilon(2 q-p)_{\mu}\right) \\
& \times \frac{q_{\rho}(p-k)_{\sigma}(p-q)_{\tau} k_{\lambda}(2 k-p)_{\nu}}{q^{4}(p-q)^{2} k^{2}}\left[\frac{1}{(k-p)^{2}}-\frac{1}{(k-p+q)^{2}}\right],
\end{aligned}
$$

Note that the original expression has no infrared divergences because of the antisymmetry of the epsilon tensors. Therefore we need not make any infrared subtractions at intermediate stages of the integration.

The contribution from the first term in parentheses yields

$$
\begin{aligned}
& \Pi_{\mu \nu}^{(d .1)}(p)= \frac{\pi^{2}}{16 \epsilon^{2}} \frac{\left[\tilde{\Gamma}_{1}(\epsilon)\right]^{2}}{\epsilon(3-2 \epsilon)(1-2 \epsilon)^{2}} \frac{1}{\left[p^{2}\right]^{2 \epsilon}} \\
& \times\left[\left(\delta_{\mu \nu}-\frac{p_{\mu} p_{\nu}}{p^{2}}\right)\left(\varepsilon^{\rho \sigma} \varepsilon_{\rho \sigma}-4 \epsilon \frac{\left(\varepsilon^{\rho \sigma} p_{\sigma}\right)^{2}}{p^{2}}\right)\right. \\
& \quad+2\left(\delta_{\mu \rho}-\frac{p_{\mu} p_{\rho}}{p^{2}}\right)\left(\delta_{\nu \sigma}-\frac{p_{\nu} p_{\sigma}}{p^{2}}\right) \varepsilon^{\rho \lambda} \varepsilon_{\lambda}^{\sigma} \\
& \\
&\left.\quad-4\left(1-2 \epsilon+2 \epsilon^{2}\right) \frac{\varepsilon_{\mu \rho} p^{\rho} \varepsilon_{\nu \sigma} p^{\sigma}}{p^{2}}\right] .
\end{aligned}
$$

This result is transverse, as it should, because this particular combination of gamma functions does not appear in any of the other graphs of Fig. 1 .

The contribution from the second term can also be determined by straightforward but somewhat tedious calculation. It reads

$$
\Pi_{\mu \nu}^{(d .2)}(p)=-\frac{\pi^{2}}{16 \epsilon^{2}} \frac{\tilde{\Gamma}_{2}(\epsilon)}{(2-3 \epsilon)(1-3 \epsilon)} \frac{1}{\left[p^{2}\right]^{2 \epsilon}}
$$




$$
\begin{gathered}
\times\left[\frac { 2 - \epsilon } { \epsilon ( 3 - 2 \epsilon ) } \left[\left(\delta_{\mu \nu}-\frac{p_{\mu} p_{\nu}}{p^{2}}\right)\left(\varepsilon^{\rho \sigma} \varepsilon_{\rho \sigma}-4 \epsilon \frac{\left(\varepsilon^{\rho \sigma} p_{\sigma}\right)^{2}}{p^{2}}\right)\right.\right. \\
\left.+2\left(\delta_{\mu \rho}-\frac{p_{\mu} p_{\rho}}{p^{2}}\right)\left(\delta_{\nu \sigma}-\frac{p_{\nu} p_{\sigma}}{p^{2}}\right) \varepsilon^{\rho \lambda} \varepsilon_{\lambda}^{\sigma}\right] \\
-\frac{4(2-3 \epsilon)(1-\epsilon)}{\epsilon(3-2 \epsilon)} \frac{\varepsilon_{\mu \rho} p^{\rho} \varepsilon_{\nu \sigma} p^{\sigma}}{p^{2}} \\
+\frac{\epsilon}{1-2 \epsilon}\left[\frac{p_{\mu} p_{\nu}}{p^{2}}\left(\varepsilon^{\rho \sigma} \varepsilon_{\rho \sigma}-2(1+2 \epsilon) \frac{\left(\varepsilon^{\rho \sigma} p_{\sigma}\right)^{2}}{p^{2}}\right)\right. \\
\left.\left.-4 \frac{p_{(\mu} \varepsilon_{\nu) \rho} \varepsilon^{\rho \sigma} p_{\sigma}}{p^{2}}\right]\right]
\end{gathered}
$$

The correctness of this result is confirmed by the fact that the (spurious) $1 / \epsilon^{3}$ pole cancels against (6.15) and that the combined result of all the graphs of Fig. 1 is gauge invariant.

The final result for the diagrams of Fig. 1 can be expressed in terms of four independent conserved structures,

$$
\begin{aligned}
\Pi_{\mu \nu}(p)=\frac{\pi^{2}}{16 \epsilon^{2}}\{ & \left(\delta_{\mu \nu}-\frac{p_{\mu} p_{\nu}}{p^{2}}\right)\left(\varepsilon^{\rho \sigma} \varepsilon_{\rho \sigma} \Pi_{1}\left(p^{2}\right)+\frac{\left(\varepsilon^{\rho \sigma} p_{\sigma}\right)^{2}}{p^{2}} \Pi_{2}\left(p^{2}\right)\right) \\
& +\left(\delta_{\mu \rho}-\frac{p_{\mu} p_{\rho}}{p^{2}}\right)\left(\delta_{\nu \sigma}-\frac{p_{\nu} p_{\sigma}}{p^{2}}\right) \varepsilon^{\rho \lambda} \varepsilon_{\lambda}^{\sigma} \Pi_{3}\left(p^{2}\right) \\
& \left.+\frac{\varepsilon_{\mu \rho} p^{\rho} \varepsilon_{\nu \sigma} p^{\sigma}}{p^{2}} \Pi_{4}\left(p^{2}\right)\right\} .
\end{aligned}
$$

Introducing the mass parameter $\mu$ the various expressions for the coefficient functions read

$$
\begin{aligned}
\Pi_{1}= & \frac{\tilde{\Gamma}_{1}(\epsilon)}{(1-\epsilon)(1-2 \epsilon)}+\frac{1}{\epsilon(3-2 \epsilon)}\left[\left[\frac{\tilde{\Gamma}_{1}(\epsilon)}{1-2 \epsilon}\right]^{2}-\frac{\tilde{\Gamma}_{2}(\epsilon)}{1-3 \epsilon}\right]\left[\frac{\mu^{2}}{p^{2}}\right]^{2 \epsilon} \\
& -\frac{4 \tilde{\Gamma}_{2}(\epsilon)}{(1+\epsilon)(1-2 \epsilon)(3-2 \epsilon)(1-3 \epsilon)}\left[\frac{\mu^{2}}{p^{2}}\right]^{2 \epsilon}, \\
\Pi_{2}= & -\frac{4}{3-2 \epsilon}\left[\left[\frac{\tilde{\Gamma}_{1}(\epsilon)}{1-2 \epsilon}\right]^{2}-\frac{\tilde{\Gamma}_{2}(\epsilon)}{1-3 \epsilon}\right]\left[\frac{\mu^{2}}{p^{2}}\right]^{2 \epsilon}-\frac{8 \epsilon \tilde{\Gamma}_{2}(\epsilon)}{(1+\epsilon)(3-2 \epsilon)(1-3 \epsilon)}\left[\frac{\mu^{2}}{p^{2}}\right]^{2 \epsilon}, \\
\Pi_{3}= & -\frac{4 \tilde{\Gamma}_{1}(\epsilon)}{1-2 \epsilon}\left[\frac{\mu^{2}}{p^{2}}\right]^{\epsilon}+\frac{2}{\epsilon(3-2 \epsilon)}\left[\left[\frac{\tilde{\Gamma}_{1}(\epsilon)}{1-2 \epsilon}\right]^{2}-\frac{\tilde{\Gamma}_{2}(\epsilon)}{1-3 \epsilon}\right]\left[\frac{\mu^{2}}{p^{2}}\right]^{2 \epsilon} \\
& -\frac{2 \tilde{\Gamma}_{2}(\epsilon)}{(1-2 \epsilon)(1-3 \epsilon)}\left[\frac{4}{(1+\epsilon)(3-2 \epsilon)}-3+2 \epsilon\right]\left[\frac{\mu^{2}}{p^{2}}\right]^{2 \epsilon}, \\
\Pi_{4}= & -\frac{8}{3}+\frac{8(2-\epsilon) \tilde{\Gamma}_{1}(\epsilon)}{3(1-2 \epsilon)}\left[\frac{\mu^{2}}{p^{2}}\right]^{\epsilon}-\frac{4\left(1-2 \epsilon+2 \epsilon^{2}\right)}{\epsilon(3-2 \epsilon)}\left[\left[\frac{\tilde{\Gamma}_{1}(\epsilon)}{1-2 \epsilon}\right]^{2}-\frac{\tilde{\Gamma}_{2}(\epsilon)}{1-3 \epsilon}\right]\left[\frac{\mu^{2}}{p^{2}}\right]^{2 \epsilon} \\
& -\frac{4(3+2 \epsilon) \tilde{\Gamma}_{2}(\epsilon)}{3(3-2 \epsilon)(1-3 \epsilon)}\left[\frac{\mu^{2}}{p^{2}}\right]^{2 \epsilon} \cdot
\end{aligned}
$$

Subsequently we evaluate $\Pi_{1}-\Pi_{4}$, up to terms of order $\epsilon^{3}$, making use of $(6.12)$. It turns out that the leading divergence (the $\epsilon$-independent term) cancels for each 
function separately and one is left with

$$
\begin{aligned}
& \Pi_{1}=-2\left(1-\ln \frac{p^{2}}{\mu^{2}}\right) \epsilon-\left(\frac{44}{3}-2 \zeta(3)-10 \ln \frac{p^{2}}{\mu^{2}}+2 \ln ^{2} \frac{p^{2}}{\mu^{2}}\right) \epsilon^{2}, \\
& \Pi_{2}=-4 \epsilon-4\left(3-2 \ln \frac{p^{2}}{\mu^{2}}\right) \epsilon^{2}, \\
& \Pi_{3}=4\left(2-\ln \frac{p^{2}}{\mu^{2}}\right) \epsilon+\left(\frac{104}{3}+4 \zeta(3)-24 \ln \frac{p^{2}}{\mu^{2}}+6 \ln ^{2} \frac{p^{2}}{\mu^{2}}\right) \epsilon^{2}, \\
& \Pi_{4}=-\left(\frac{16}{3}+8 \zeta(3)-8 \ln \frac{p^{2}}{\mu^{2}}+\frac{8}{3} \ln ^{2} \frac{p^{2}}{\mu^{2}}\right) \epsilon^{2} .
\end{aligned}
$$

As we shall discuss in the next subsection, also the next-to-leading divergence cancels, as well as the Riemann $\zeta(3)$ function, once these terms are combined, when the components and momenta of the external current are restricted to two dimensions.

\subsection{Subdivergences and evanescent counterterms}

In the previous subsection we ignored the presence of ultraviolet divergences in some of the one-loop subgraphs. In principle these subdivergences, which involve the quantum fields, must be consistently removed by means of counterterms. Since the subgraphs do not necessarily involve the background fields, these counterterms are not contained in our previous one-loop calculations and must be determined separately. This issue turns out to be related to the way in which one deals with the epsilon tensor.

It is well known that the naive continuation away from two dimensions for products of the epsilon tensor,

$$
\varepsilon_{\mu \nu} \varepsilon^{\rho \sigma}=f(\epsilon)\left(\delta_{\mu}^{\rho} \delta_{\nu}^{\sigma}-\delta_{\nu}^{\rho} \delta_{\mu}^{\sigma}\right)
$$

with $f(0)=1$ and $\delta_{\mu}^{\nu}$ the $d$-dimensional Kronecker symbol, becomes inconsistent at dimensions different from two when products of more than two epsilon tensors are considered [4]. Furthermore its consequences are in disagreement with general results, both for the WZNW model away from the critical point [4 and for twodimensional sigma models with torsion [5]. The generally accepted rule is to keep the epsilon tensors in two dimensions, so that the tensors $\delta_{\mu}^{\nu}$ on the right-hand side of (6.20) should be replaced by two-dimensional Kronecker symbols and $f(\epsilon)=1$. Interestingly enough, provided subdivergences are treated properly, both two- and $d$ dimensional epsilon tensors lead to the same two-loop result, as we will demonstrate in this subsection.

We consider first the treatment of the epsilon tensors as $d$-dimensional objects. Although the rule (6.20) is not consistent for products of more than two epsilon tensors, we might still accept it in evaluating our result as our expressions involve only two such tensors. For the contributions of the previous subsection, which are given in (6.17-19), this yields the following,

$$
\frac{\pi^{2}}{16 \epsilon^{2}}\left\{2(1-\epsilon) \Pi_{1}+\Pi_{2}+\Pi_{3}+\Pi_{4}\right\}=\pi^{2}\left(-\frac{1}{2}+\frac{1}{2} \ln \frac{p^{2}}{\mu^{2}}-\frac{1}{24} \ln ^{2} \frac{p^{2}}{\mu^{2}}\right)+\mathcal{O}(\epsilon)
$$


multiplied by $\left(\delta_{\mu \nu}-p_{\mu} p_{\nu} / p^{2}\right)$. Combining this with (6.6) leads directly to the result quoted in (6.2).

At the same time it can be argued that the explicit subtraction of the one-loop divergences is unnecessary, since they cancel amongst themselves. This is so because the corresponding counterterms are proportional to the Lagrangian (3.11) (because of background gauge invariance and manifest rotational invariance in $d$ dimensions) and correspond to a multiplicative renormalization of the quantum fields. However, diagrams with only external background fields are insensitive to the normalization of the quantum fields. Therefore the counterterms corresponding to this wave-function renormalization do not contribute, so that the result quoted in (6.2) represents the full two-loop contribution.

Instead we consider keeping the epsilon tensor in precisely two dimensions. By doing so we lose manifest rotational invariance in $d$ dimensions and the counterterms no longer have the same form as the original Lagrangian. In our calculation only part of the divergences can be absorbed by a multiplicative renormalization of the quantum fields. The remaining divergences are of the "evanescent" type, i.e. they are proportional to operators that vanish for $d=2$. They must be subtracted by additional counterterms (even at the critical point), which will give rise to finite contributions to our result. At the same time the result from the graphs of subsection 6.2 is different, although still finite, when evaluated for two-dimensional epsilon tensors. We obtain from (6.17-19),

$$
\frac{\pi^{2}}{16 \epsilon^{2}}\left\{2 \Pi_{1}+\Pi_{2}+\Pi_{3}+\Pi_{4}\right\}=\pi^{2}\left(-\frac{3}{4}+\frac{3}{4} \ln \frac{p^{2}}{\mu^{2}}-\frac{1}{24} \ln ^{2} \frac{p^{2}}{\mu^{2}}\right)+\mathcal{O}(\epsilon)
$$

multiplied again by $\left(\delta_{\mu \nu}-p_{\mu} p_{\nu} / p^{2}\right)$. Here we assumed that the indices and momenta of the external currents were restricted to two dimensions. The purpose of the discussion below is to demonstrate that the difference between this result and the previous one is precisely compensated for by the evanescant part of the subtractions related to subgraphs.

The divergent subgraphs consist of the diagrams with two external quantum fields and up to two background currents. From power counting and background gauge invariance we know that the divergences take the form of the Lagrangian (3.10), except that the space-time metric is not necessarily equal to $\delta^{\mu \nu}$, but can be decomposed into $\delta^{\mu \nu}$ and $\hat{\hat{\delta}}^{\mu \nu}$, where the latter takes its value only in the $d-2$ extra dimensions. The contribution of this tensor thus leads to an evanescent operator, which, as it is proportional to a $1 / \epsilon$ pole, must be subtracted by an appropriate counterterm. As explained above the divergences proportional to $\delta^{\mu \nu}$ do not contribute to the result of our calculation as they just correspond to a multiplicative renormalization of the quantum fields.

Hence we are left with the evanescent counterterm. As the components and the momenta of the background currents are restricted to two dimensions, their contribution can be ignored and we concentrate on the counterterm quadratic in the quantum fields, which comes from the self-energy graphs with vertices from the first term in (3.13) proportional to the epsilon tensor. A simple calculation reveals 
the need for the following counterterm,

$$
\Delta \mathcal{L}=-\frac{\tilde{h}}{32 \pi \epsilon} \hat{\hat{\delta}}^{\mu \nu} \partial_{\mu} \vec{\pi} \cdot \partial_{\nu} \vec{\pi}
$$

Further finite parts in $\Delta \mathcal{L}$ proportional to $\hat{\hat{\delta}}^{\mu \nu}$ will not contribute, as the pole term in $\Delta \mathcal{L}$ gives already a finite result in the final answer.

This evanescent counterterm must be inserted into the one-loop diagrams. These are the diagrams evaluated in section 4 (cf. (4.1)) and we find

$$
\Delta \Pi_{\mu \nu}^{a b}(p)=\tilde{h} g^{a b} \int \frac{\mathrm{d}^{2} k}{(2 \pi)^{2}}\left[\frac{(2 k-p)_{\mu}(2 k-p)_{\nu}}{k^{4}(p-k)^{2}}-\frac{\delta_{\mu \nu}}{k^{4}}\right]\left[-k_{\rho} k_{\sigma} \hat{\hat{\delta}}^{\rho \sigma} \frac{\tilde{h}}{16 \pi \epsilon}\right] \frac{8 \pi}{k}
$$

where the factor $8 \pi / k$ arises because the evanescent counterterm is not proportional to $k / 8 \pi$, in contrast to all other terms in the Lagrangian. The integral can easily be evaluated with the formulae given in the appendix. The calculation simplifies considerably because the momentum $p$ and the indices $\mu$ and $\nu$ are kept two-dimensional. After subtracting the infrared divergences, as before, we find the result

$$
\Delta \Pi_{\mu \nu}(p)=-\frac{\pi^{2}}{4 \epsilon}\left[\frac{1}{1-\epsilon}-\frac{\Gamma(1+\epsilon)[\Gamma(1-\epsilon)]^{3}}{\Gamma(2-2 \epsilon)}\left[\frac{\mu^{2}}{p^{2}}\right]^{\epsilon}\right]\left(\delta_{\mu \nu}-\frac{p_{\mu} p_{\nu}}{p^{2}}\right) .
$$

Here we extracted an overall factor according to (6.1). Observe that the answer is again transverse. It is also finite, as a result of the subtraction of infrared divergences.

Expanding the above result in powers of $\epsilon$ we derive

$$
\Delta \Pi_{\mu \nu}(p)=\frac{1}{4} \pi^{2}\left(1-\ln \frac{p^{2}}{\mu^{2}}\right)\left(\delta_{\mu \nu}-\frac{p_{\mu} p_{\nu}}{p^{2}}\right) .
$$

Adding this result to (6.22) and (6.6) leads directly to the result quoted in (6.2) and confirms our claim that a proper treatment with two-dimensional epsilon tensors gives the same as the calculation based on $d$-dimensional epsilon tensors, at least in this order of perturbation theory. 


\section{Conclusions}

In this paper we have studied the gauge-invariant action (3.10) with normal group coordinates $h=\exp \left(\pi^{a} T_{a}\right)$ as quantum fields and background fields $J_{+}=\partial_{+} g g^{-1}$ and $J_{-}=\partial_{-} \bar{g} \bar{g}^{-1}$. Using this action we computed the one-particle irreducible Green's function of two currents in the two-loop approximation. Although we were primarily interested in the WZNW model at the conformal point, our calculations apply as well away from the critical point because we have kept separate the contributions with and without the $\varepsilon^{\mu \nu}$ tensors. Off the critical point, the one- and two-loop contribution to the two-point function is

$$
\begin{aligned}
& \Pi_{\mu \nu}^{a b}(p)=\frac{\tilde{h}}{2 \pi} g^{a b}\left(\delta_{\mu \nu}-\frac{p_{\mu} p_{\nu}}{p^{2}}\right)\left\{1-\frac{1}{2} \ln \frac{p^{2}}{\mu^{2}}+\frac{1}{12} \frac{\tilde{h}}{k}\left(\frac{k g^{2}}{2 \pi}\right) \ln ^{2} \frac{p^{2}}{\mu^{2}}\right. \\
&\left.+\frac{1}{2} \frac{\tilde{h}}{k}\left(\frac{k g^{2}}{2 \pi}\right)\left[1-\left(\frac{k g^{2}}{2 \pi}\right)^{2}\right]\left(1-\ln \frac{p^{2}}{\mu^{2}}+\frac{1}{12} \ln ^{2} \frac{p^{2}}{\mu^{2}}\right)\right\} .
\end{aligned}
$$

This expression may be viewed as a quantum correction to the classical correlation functions described by $S_{W}^{+}\left[\bar{g}^{-1} g\right]$, as follows from the splitting (cf. (3.7))

$$
S_{W}^{+}\left[\bar{g}^{-1} h g\right]=S_{W}^{+}\left[\bar{g}^{-1} g\right]+S[\vec{\pi}, \vec{J}] .
$$

In particular, at the critical point, one finds, after omitting the logarithmic terms in (7.1), that our result is consistent with the following renormalization

$$
S_{W}^{+}\left[\bar{g}^{-1} g\right] \longrightarrow S_{\mathrm{eff}}[\vec{J}]=\frac{k+\tilde{h}}{k} S_{W}^{+}\left[\bar{g}^{-1} g\right] .
$$

Here the symbol $S_{\text {eff }}$ denotes the sum of the one-particle irreducible graphs with external currents $J$ and no external quantum fields computed with the action (3.10) while $S_{W}^{+}\left[\bar{g}^{-1} g\right]$ is the covariant induced Yang-Mills action (cf. (2.16)).

To derive this result we used dimensional regularization to deal with both ultraviolet and infrared divergences. As stressed repeatedly, the result is free of ultraviolet divergences, while the infrared divergences were removed by "minimal" subtractions using the $R^{*}$ scheme. We believe, although we know of no actual proof, that this procedure preserves gauge invariance. The ambiguity associated with infrared subtractions is reflected in the occurence of a scale parameter $\mu$ in the logarithms in (7.1). Apart from this, our results do not depend on the particular regularization scheme employed as long as it preserves gauge invariance since different schemes can only yield differences by local counterterms (of dimension 2 or less). However, there are no gauge-invariant local counterterms constructed from the background currents of dimension 2. This is the reason why using $d$-dimensional epsilon tensors gives the same as two-dimensional epsilon symbols, provided we treat subdivergences correctly. These considerations hold quite generally both off and on the critical point. Off the critical point gauge invariance implies ultraviolet finitenes of $S_{\text {eff }}(J)$ provided one includes counterterms for the coupling constant renormalization (the counterterms for the wave function renormalization are not needed in Green's functions without external quantum fields). At the critical point the coupling constant does 
not renormalize. In that case one only need subtract infrared subdivergences (and possibly evanescent counterterms). At any rate, as is clear from (7.1), coupling constant renormalization will only enter beyond the two-loop approximation. (This in contradistinction with the connected current correlation functions, which require a coupling constant renormalization already at the one-loop level, as demonstrated in subsection 4.4.)

The shift $k \rightarrow k+\tilde{h}$ in (7.2) is familiar from conformal field theory, where it appears both in the quantum field equation for the group-valued field $g$ and in the stress tensor [6]. However, in conformal field theory one considers connected graphs whereas the effective action we have been computing consists of one-particle irreducible graphs for which techniques of conformal field theory are not directly applicable. To the best of our knowledge, no formal proof exists that the effective action of this paper must exhibit a shift of the level by an integer. On the other hand the result is reasonable, as it corresponds to the action induced by real bosons. Therefore one does expect the same induced action as for fermions (in the same representation of the gauge group) but with a relative factor -2 . However, the fact that we find vanishing contributions (up to logarithmic terms) beyond one loop is highly nontrivial and finds its origin in the underlying structure of the WZNW model. As we already discussed in subsection 4.1, the infrared divergences and the logarithmic corrections that they induce are due to the fact that the fields $\vec{\pi}$, unlike fermion fields, are not primary.

At this point we should clarify the relation between our action and other actions that have appeared in the literature. We distinguish four actions which are all different off the critical point but which are related in various ways at the critical point:

1. The classical action $S[\vec{\pi}, \vec{J}]$ we have been using in this article (and its extension off the critical point), describing the gauged WZNW model. Quantum corrections lead to an effective action describing the one-particle irreducible current correlation functions. Gauge invariance guarantees the finiteness of this effective action both on and off the critical point.

2. The standard action of ordinary quantum field theory obtained from (3.15) by putting $g=\exp \pi^{a} T_{a}$. For $k g^{2}=2 \pi$ one has the WZNW model, which is classically equivalent to the induced action for Yang-Mills fields coupled to matter as we discussed in section 2. In general, as sketched in subsection 4.4, the corresponding effective action has divergences, requiring only wave-function renormalization at the critical point, but also coupling constant renormalization (non-vanishing $\beta$-function) away from the critical point.

3. The background field action used by Witten and Bos to extract the coupling constant renormalization [3, 1 . It follows from (3.15), replacing $g$ by $h g$, and treating $h$ as a quantum field and $g$ as a background field. This leads to a coupling to the current

$$
J_{\mu} \propto \frac{2 \pi}{k g^{2}} \partial_{\mu} g g^{-1}-i \varepsilon_{\mu \nu} \partial^{\nu} g g^{-1}
$$


This current couples differently than the current in our action. Consequently, away from the critical point, the cancellations of ultraviolet divergences implied by gauge invariance do not occur. For example, at the one-loop level the second term in the integral of (4.1) is absent (cf. eq. (12) of [4]). At the critical point the resulting effective action is, however, finite and coincides with our effective action restricted to the chiral sector with $J_{-}=0$.

4. The action considered by Leutwyler and Shifman [8]. It is given by (3.15) with $g$ replaced by $\bar{g}^{-1} h g$, and treating $g$ and $\bar{g}$ as background fields. Away from the critical point it leads to two currents (associated with the invariance under right and left multiplications of the group-valued field),

$$
\begin{aligned}
& J_{\mu} \propto \frac{2 \pi}{k g^{2}} \partial_{\mu} g g^{-1}-i \varepsilon_{\mu \nu} \partial^{\nu} g g^{-1} \\
& \bar{J}_{\mu} \propto \frac{2 \pi}{k g^{2}} \partial_{\mu} \bar{g} \bar{g}^{-1}+i \varepsilon_{\mu \nu} \partial^{\nu} \bar{g} \bar{g}^{-1} .
\end{aligned}
$$

The action is not invariant under independent local gauge transformations of the two currents At the critical point the currents are restricted to (anti)selfdual vectors and this action and ours coincide. Off the critical point the diagonal truncation $g=\bar{g}$ expresses the two currents in terms of a single current $\partial_{\mu} g g^{-1}$ and the action coincides again with our action. Obviously the truncation $\bar{g}=\mathbf{1}$ leads to the action considered in [3, 团.

Thus, it seems that our effective action is a reasonable object on which to base further studies, at least at the critical point. Its full gauge invariance is very helpful in controlling details of the calculations, and its restriction to the chiral sector $J_{-}=0$ reproduces the usual background field effective action. Furthermore, it gives the oneparticle irreducible part of the full current correlation function. Finally, it exhibits the standard shift $k \rightarrow k+\tilde{h}$, with no two-loop correction.

We have computed in the one-loop approximation the two- and three-point functions for the induced action. While this theory is classically equivalent to the WZNW theory, its short-distance behaviour is qualitatively worse. Although the results of the calculations are still finite due to Lorentz invariance, this behaviour leads to ambiguities in the final results. These ambiguities are fixed by using a specific rule for the exponential regulators, as described after (5.9) and also in [20]. Our result, given in (5.21), agrees with that obtained by semiclassical methods [10, 17] based directly on the anomalous Ward identity (2.11),

$$
\Gamma_{i n d}\left[\overrightarrow{\mathcal{A}}_{ \pm}\right]=Z_{k} S_{i n d}^{ \pm}\left[Z_{A} \overrightarrow{\mathcal{A}}_{ \pm}\right], \text {with } Z_{k}=\frac{k-2 \tilde{h}}{k}, \quad Z_{A}=1+\frac{\tilde{h}}{k}
$$

where $\Gamma_{\text {ind }}\left[\overrightarrow{\mathcal{A}}_{ \pm}\right]$is the effective action defined below (cf. (7.8)). As far as the level shift is concerned we also agree with the result of a recent calculation 18 based on Pauli-Villars regularization. However, the field renormalization takes a different value (which depends on the presence of certain counterterms that are motivated by symmetry arguments). Although it is sometimes claimed that the subtraction dependence reflects itself solely in the field renormalization (see e.g. [9]), we know 
of no rigorous proof that the level shift should be independent of the regularization procedure. There exists a variety of formal arguments leading to different values for the renormalization constants (both the shift and the field-renormalization factor) and a regularization scheme that makes the formal manipulations underlying one such derivation rigorous will lead to these renormalization constants (unless there is some inconsistency within this set of manipulations). Our calculation in section 5 neither proves nor disproves the above claim.

One might hope that by relating the induced theory to the WZNW theory, one could somehow resolve these ambiguities. At the quantum level the relation hinges on the Jacobian associated with the change of integration variables $A_{ \pm}=$ $\partial_{ \pm} g g^{-1}$ in the path integral. This Jacobian is formally related to a chiral fermionic determinant, which in turn defines the induced action. In section 4 we compared the two- and three-point function for the WZNW theory to that of a chiral fermion theory, confirming the relation of the fermionic determinant to the induced action. So it seems justified to assume the following relation

$$
\mathcal{D} A_{ \pm}=\exp \left(\frac{-2 \tilde{h}}{k} S_{W}^{ \pm}[g]\right) \mathcal{D} g
$$

Inserting this result into (4.20), we relate the generating functional for the connected current correlation functions in the WZNW theory to the corresponding quantity for the induced action, defined by

$$
\exp W_{i n d}^{\mp}\left[J_{\mp}\right]=\int \mathcal{D} A_{ \pm} \exp \left(S_{i n d}^{ \pm}\left[\vec{A}_{ \pm}\right]+\frac{k \lambda^{2}}{4 \pi} \int \mathrm{d}^{2} x \vec{J}_{\mp} \cdot \vec{A}_{ \pm}\right) .
$$

but now with level $k+2 \tilde{h}$ and rescaled source $\vec{J}_{\mp}$. However, the generating functional for the WZNW theory is not renormalized (cf. (4.21)). In this way we establish that $W_{\text {ind }}^{\mp}\left[\vec{J}_{\mp}\right]$ is equal to

$$
W_{\text {ind }}^{\mp}\left[\vec{J}_{\mp}\right]=-Z_{k} S_{\text {ind }}^{\mp}\left[Z_{J} \vec{J}_{\mp}\right], \text { with } Z_{k}=\frac{k-2 \tilde{h}}{k}, \quad Z_{J}=\frac{k}{k-2 \tilde{h}} .
$$

The corresponding effective action, defined by a Legendre transform,

$$
\Gamma_{i n d}^{ \pm}\left[\overrightarrow{\mathcal{A}}_{ \pm}\right]=W_{i n d}\left[\vec{J}_{ \pm}\right]-\frac{k \lambda^{2}}{4 \pi} \int \mathrm{d}^{2} x \vec{J}_{\mp} \cdot \overrightarrow{\mathcal{A}}_{ \pm}, \quad \text { with } \quad \overrightarrow{\mathcal{A}}_{ \pm} \equiv \frac{\partial W_{i n d}^{\mp}\left[\vec{J}_{\mp}\right]}{\partial \vec{J}_{\mp}}
$$

\footnotetext{
${ }^{4}$ Most formal derivations lead to the same level shift (for a discussion, see [17, 13, 15, 18]). A formal proof that the effective action remains unrenormalized (no level shift) follows from the observation that

$$
\delta F\left[A_{ \pm}\right]=\int \mathrm{d}^{2} x\left(\partial_{ \pm} \vec{\eta}(x)+\vec{\eta}(x) \times \vec{A}_{ \pm}(x)\right)^{a} \frac{\partial F\left[A_{ \pm}\right]}{\partial A_{ \pm}^{a}(x)},
$$

where $F\left[A_{ \pm}\right]$is an arbitrary functional, can be written as a total derivative with respect to $\vec{A}$ (we assume a semisimple group, so that $f_{a b}^{a}=0$ ). Therefore it follows that $\int \mathcal{D} A_{ \pm} \delta F\left[A_{ \pm}\right]=0$. Using this result in the functional integral that defines the generating functional $W_{\text {ind }}^{\mp}\left[\vec{J}_{\mp}\right]$ for the connected Green's functions (cf. (7.6)), one proves that this functional satisfies the anomalous Ward identity $(2.11)$ with opposite sign. Therefore it follows that $W_{\text {ind }}^{\mp}\left[\vec{J}_{\mp}\right]=-S_{\text {ind }}^{\mp}\left[\vec{J}_{\mp}\right]$ and the effective action equals $\Gamma_{\text {ind }}^{ \pm}\left[\overrightarrow{\mathcal{A}}_{\mp}\right]=S_{\text {ind }}^{ \pm}\left(\overrightarrow{\mathcal{A}}_{ \pm}\right)$.
} 
can be evaluated by means of (2.16). We find

$$
\Gamma_{i n d}^{ \pm}\left[\overrightarrow{\mathcal{A}}_{ \pm}\right]=Z_{k} S_{i n d}^{ \pm}\left[\overrightarrow{\mathcal{A}}_{ \pm}\right]
$$

Hence the multiplicative renormalization for the field is now absent, while the level shift $k \rightarrow k-2 \tilde{h}$ is placed on firmer ground. Nevertheless, while the above argument, due to [13], does indeed give a relation between the effective action of the induced theory and the (connected) current correlation functions of the WZNW theory, further work confronting formal arguments with explicit calculations is needed.

Acknowledgements: We thank J. de Boer, J. Goeree, A. Sevrin, R. Siebelink, W. Troost, A. Tseytlin, and D. Zanon for valuable discussions.

\section{A Useful formulae}

The following formulae can be derived by differentiation of the basic formula (4.4) with respect to the momentum $p$,

$$
\begin{aligned}
& \Gamma(1-\epsilon)(4 \pi)^{-\epsilon} \int \frac{\mathrm{d}^{d} k}{(2 \pi)^{d}} \frac{k_{\mu}}{\left[k^{2}\right]^{\alpha}\left[(k-p)^{2}\right]^{\beta}} \\
& =\frac{1}{4 \pi} \frac{\Gamma(1-\epsilon) \Gamma(\alpha+\beta-1+\epsilon) \Gamma(2-\epsilon-\alpha) \Gamma(1-\epsilon-\beta)}{\Gamma(\alpha) \Gamma(\beta) \Gamma(3-2 \epsilon-\alpha-\beta)} \frac{p_{\mu}}{\left[p^{2}\right]^{\alpha+\beta-1+\epsilon}}, \\
& \Gamma(1-\epsilon)(4 \pi)^{-\epsilon} \int \frac{\mathrm{d}^{d} k}{(2 \pi)^{d}} \frac{k_{\mu} k_{\nu}}{\left[k^{2}\right]^{\alpha}\left[(k-p)^{2}\right]^{\beta}} \\
& =\frac{1}{8 \pi} \frac{\Gamma(1-\epsilon) \Gamma(\alpha+\beta-2+\epsilon) \Gamma(2-\epsilon-\alpha) \Gamma(2-\epsilon-\beta)}{\Gamma(\alpha) \Gamma(\beta) \Gamma(4-2 \epsilon-\alpha-\beta)} \frac{1}{\left[p^{2}\right]^{\alpha+\beta-2+\epsilon}} \\
& \times\left\{\delta_{\mu \nu}-\frac{2(2-\alpha-\beta-\epsilon)(2-\alpha-\epsilon)}{1-\beta-\epsilon} \frac{p_{\mu} p_{\nu}}{p^{2}}\right\}, \\
& \Gamma(1-\epsilon)(4 \pi)^{-\epsilon} \int \frac{\mathrm{d}^{d} k}{(2 \pi)^{d}} \frac{k_{\mu} k_{\nu} k_{r}}{\left[k^{2}\right]^{\alpha}\left[(k-p)^{2}\right]^{\beta}} \\
& =\frac{1}{8 \pi} \frac{\Gamma(1-\epsilon) \Gamma(\alpha+\beta-2+\epsilon) \Gamma(3-\epsilon-\alpha) \Gamma(2-\epsilon-\beta)}{\Gamma(\alpha) \Gamma(\beta) \Gamma(5-2 \epsilon-\alpha-\beta)} \frac{1}{\left[p^{2}\right]^{\alpha+\beta-2+\epsilon}} \\
& \times\left\{3 p_{(\mu} \delta_{\nu \rho)}-\frac{2(2-\alpha-\beta-\epsilon)(3-\alpha-\epsilon)}{1-\beta-\epsilon} \frac{p_{\mu} p_{\nu} p_{\rho}}{p^{2}}\right\} \frac{1}{\left[p^{2}\right]^{\alpha+\beta-2+\epsilon}}, \\
& \Gamma(1-\epsilon)(4 \pi)^{-\epsilon} \int \frac{\mathrm{d}^{d} k}{(2 \pi)^{d}} \frac{k_{\mu} k_{\nu} k_{\rho} k_{\sigma}}{\left[k^{2}\right]^{\alpha}\left[(k-p)^{2}\right]^{\beta}} \\
& =\frac{1}{16 \pi} \frac{\Gamma(1-\epsilon) \Gamma(\alpha+\beta-3+\epsilon) \Gamma(3-\epsilon-\alpha) \Gamma(3-\epsilon-\beta)}{\Gamma(\alpha) \Gamma(\beta) \Gamma(6-2 \epsilon-\alpha-\beta)} \frac{1}{\left[p^{2}\right]^{\alpha+\beta-3+\epsilon}} \\
& \times\left\{3 \delta_{(\mu \nu} \delta_{\rho \sigma)}-\frac{12(3-\alpha-\beta-\epsilon)(3-\alpha-\epsilon)}{2-\beta-\epsilon} \frac{\delta_{(\mu \nu} p_{\rho} p_{\sigma)}}{p^{2}}\right.
\end{aligned}
$$




$$
\left.+\frac{4(3-\alpha-\beta-\epsilon)(2-\alpha-\beta-\epsilon)(3-\alpha-\epsilon)(4-\alpha-\epsilon)}{(2-\beta-\epsilon)(1-\beta-\epsilon)} \frac{p_{\mu} p_{\nu} p_{\rho} p_{\sigma}}{p^{4}}\right\},
$$

where, as before, we inserted the $\Gamma(1-\epsilon)(4 \pi)^{-\epsilon}$ factor to avoid extraneous quantities such as the Euler constant $\gamma_{E}$ or the Riemann $\zeta(2)$ function. In the above equation the symmetrization over vector indices is with strength one.

For the convenience of the reader we also add the following expansion formula for the gamma function

$$
\ln \Gamma(1+z)=-\gamma_{E} z+\frac{1}{12} \pi^{2} z^{2}-\frac{1}{3} \zeta(3) z^{3}+\mathcal{O}\left(z^{4}\right)
$$

We now discuss some features of the infrared subtraction procedure for two-loop diagrams (see also the appendix of [30]). Beyond the one-loop level we encounter non-integer powers of propagators. The required subtraction rule is a modification of $(4.3)$,

$$
\frac{1}{\left[k^{2}\right]^{1+\tau \epsilon}} \rightarrow \frac{1}{\left[k^{2}\right]^{1+\tau \epsilon}}+\frac{\pi}{(1+\tau) \epsilon} \delta^{(2)}(k)
$$

A related rule is

$$
\frac{k_{\mu} k_{\nu}}{\left[k^{2}\right]^{2+\tau \epsilon}} \rightarrow \frac{k_{\mu} k_{\nu}}{\left[k^{2}\right]^{2+\tau \epsilon}}+\frac{\delta_{\mu \nu}}{2(1+\tau)} \frac{\pi}{\epsilon(1-\epsilon)} \delta^{(2)}(k) .
$$

As stressed in section 4, the subtraction scheme works exactly as the BPHZ scheme for ultraviolet divergences. Rather than discussing the general formulation (for that we refer to [25]), we illustrate the procedure for the diagram 4.b, where the infrared divergences are overlapping. The corresponding integral is

$$
I_{\rho \sigma}=\int \mathrm{d}^{2} k \mathrm{~d}^{2} q \frac{q_{\rho}(k-2 q)_{\sigma}}{q^{2}(k-q)^{2}(k-p)^{2}}
$$

We consider first infrared divergent subdiagrams consisting of single propagators. For each propagator we subtract $-\pi / \epsilon \delta^{(2)}(p)$. In principle, this generates three terms . Here the subtraction at $q=0$ vanishes, and one is left with two single integrals,

$$
\frac{\pi}{\epsilon} \int \mathrm{d}^{2} k \frac{-k_{\rho} k_{\sigma}}{k^{2}(k-p)^{2}} \quad \text { and } \quad \frac{\pi}{\epsilon} \int \mathrm{d}^{2} q \frac{q_{\rho}(p-2 q)_{\sigma}}{q^{2}(q-p)^{2}},
$$

corresponding, respectively, to poles at $q=k$ and $k=p$.

Subsequently we subtract the infrared divergences associated with one-loop subdiagrams. In this case there are three one-loop subdiagrams. We calculate each one of them, including their proper infrared subtractions associated with the lowestorder propagators, so that the combined result is finite. Then, using (A.6-7), we

\footnotetext{
${ }^{5}$ We consider diagrams with only a single subtraction. There are also four terms with multiple subtractions (i.e. on two or three different propagators). However, the multiple subtractions can be ignored as they are again contained in the subtractions (discussed below) associated with higher-loop subdiagrams.
} 
determine the corresponding overall infrared counterterm. For (A.6) we must extract only the pole term, taking the limit $\epsilon \rightarrow 0$ in the residue, while for (A.7), we must retain the factor $1 /(1-\epsilon)$ in order that (A.7) be compatible with (A.6).

We now consider the three subdiagrams in detail. The first one contains the propagators with momenta $q$ and $p-k$. We extract the third propagator $(k-q)^{-2}$ and obtain (after relabeling $q \rightarrow k-q$ ),

$$
\frac{1}{q^{2}}\left\{\int \mathrm{d}^{2} k \frac{(k-q)_{\rho}(-k+2 q)_{\sigma}}{(k-q)^{2}(k-p)^{2}}+\frac{\pi}{\epsilon} \frac{(p-q)_{\rho}(-p+2 q)_{\sigma}}{(p-q)^{2}}\right\} .
$$

The second subdiagram contains the propagators with momenta $q$ and $k-q$. After extracting the third propagator $(k-p)^{-2}$ we obtain

$$
\frac{1}{(k-p)^{2}}\left\{\int \mathrm{d}^{2} q \frac{q_{\rho}(k-2 q)_{\sigma}}{q^{2}(k-q)^{2}}-\frac{\pi}{\epsilon} \frac{k_{\rho} k_{\sigma}}{k^{2}}\right\} .
$$

The third subdiagram contains the propagators with momenta $p-k$ and $k-q$. Extracting a factor $q_{\rho} q^{-2}$ we obtain

$$
\frac{q_{\rho}}{q^{2}}\left\{\int \mathrm{d}^{2} k \frac{(k-2 q)_{\sigma}}{(k-q)^{2}(k-p)^{2}}+\frac{\pi}{\epsilon} \frac{(p-3 q)_{\sigma}}{(p-q)^{2}}\right\} .
$$

The above three one-loop subdiagrams are themselves infrared finite but may lead to an infrared divergence when inserted into the final integral. To subtract this divergence we must add corresponding counterterms. One easily verifies that only the third subdiagram requires such a counterterm. The integral in (A.12) gives (replacing $k_{\sigma}$ in the numerator by $\frac{1}{2}(p+q)_{\sigma}$ )

$$
\int \mathrm{d}^{2} k \frac{\frac{1}{2}(p-3 q)_{\sigma}}{(k-q)^{2}(k-p)^{2}} \longrightarrow-\frac{\pi}{\epsilon} \frac{[\Gamma(1-\epsilon)]^{3} \Gamma(1+\epsilon)}{\Gamma(1-2 \epsilon)} \frac{(p-3 q)_{\sigma}}{\left[(p-q)^{2}\right]^{1+\epsilon}} .
$$

The counterterm is obtained by applying (A.6) both to (A.13) (retaining only the $1 / \epsilon$ pole) and to the second term in (A.12). The combined infrared counterterm, to be inserted into the final integral over $q$, equals

$$
\frac{\pi^{2}}{2 \epsilon^{2}}(p-3 q)_{\sigma} \delta^{(2)}(p-q)
$$

multiplied by $q_{\rho} q^{-2}$ (cf.(A.12)).

Assembling all the pieces together leads to the first term quoted in (6.9). 


\section{References}

[1] J. Wess and B. Zumino, Phys. Lett. 37B (1971) 95

[2] S.P. Novikov, Sov. Math. Doklady 24 (1981) 222; Usp. Math. Nauk. 37 (1982) 3

[3] E. Witten, Nucl. Phys. B223 (1983) 422; Commun. Math. Phys. 92 (1984) 455

[4] M. Bos, Phys. Lett. B189 (1987) 435; Ann. Phys. (N.Y.) 181 (1988) 177.

[5] S. Ketov, Nucl. Phys. B294 (1987) 813;

C. Hull and P.K. Townsend, Phys. Lett. 191B (1987) 115;

R.R. Metsaev and A.A. Tseytlin, Phys. Lett. 191B (1987) 354;

D. Zanon, Phys. Lett. 191B (1987) 363;

D.R.T. Jones, Phys. Lett. 192B (1987) 391.

[6] V.G. Knizhnik and A.B. Zamolodchikov, Nucl. Phys. B247 (1984) 83

[7] A.M. Polyakov and P.B. Wiegmann, Phys. Lett. 131B (1983) 121; 141B (1984) 223.

[8] H. Leutwyler and M. Shifman, Int. J. Mod. Phys. A7 (1992) 795.

[9] A. Tseytlin, preprints Imperial/TP/92-93/10 and CERN-TH.6804/93.

[10] A.M. Polyakov, in Fields, Strings and Critical Phenomena, Les Houches 1988, E. Brézin and J. Zinn-Justin (eds.), North-Holland, 1990.

[11] H. Ooguri, K. Schoutens, A. Sevrin and P. van Nieuwenhuizen, Commun. Math. Phys. 145 (1992) 515.

[12] M. Bershadsky and H. Ooguri, Commun. Math. Phys. 126 (1989) 49; Phys. Lett. 229B (1989) 374.

[13] J. de Boer and J. Goeree, The effective action of $W_{3}$ gravity to all orders, Nucl. Phys. B, in print.

[14] G.W. Delius, M.T. Grisaru and P. van Nieuwenhuizen, Nucl. Phys. B389 (1993) 25.

[15] A. Sevrin, K. Thielemans and W. Troost, preprint LBL-33738, KUL-TF-93/09.

[16] K. Schoutens, A. Sevrin and P. van Nieuwenhuizen, Nucl. Phys. B364 (1991) 584; B371 (1992) 315.

[17] K. Schoutens, A. Sevrin and P. van Nieuwenhuizen, in Strings 83 Symmetries 1991, proc. of the Stony Brook conference, eds N. Berkovits et al., World Scientific, 1992.

[18] A. Sevrin, R. Siebelink and W. Troost, Leuven preprint, in preparation.

[19] A.M. Polyakov, Gauge fields and strings, Harwood, 1987. 
[20] M.T. Grisaru and P. van Nieuwenhuizen, Int. J. Mod. Phys. A7 (1992) 5891.

[21] E. Guadagnini, M. Martellini and M. Mintchev, Phys. Lett. B227 (1989) 111; W. Chen, G.W. Semenoff and Yong-Shi Wu, Mod. Phys. Lett. A5 (1990) 1833; C.P. Martin, Phys. Lett. B241 (1990) 513;

D. Birmingham, M. Rakowski and G. Thomson, Nucl. Phys. B329 (1990) 83;

L. Alvarez-Gaumé, J.M.F. Labastida and A.V. Ramallo, Nucl. Phys. B334 (1990) 103 ;

M. Shifman, Nucl. Phys. B352 (1991) 87;

C. Imbimbo, Phys. Lett. 258B (1991) 353.

[22] K. Bardakci, E. Rabinovici and B. Säring, Nucl. Phys. B299 (1988) 157;

K. Gawedzki and A. Kupiainen, Phys. Lett. B215 (1988) 119; Nucl. Phys. B320 (1989) 625;

D. Karabali, Q-Han Park, H.J. Schnitzer and Z. Yang, Phys. Lett. B216 (1989) 625.

[23] K.A. Meisner and J. Pavelchik, Mod. Phys. Lett. A5 (1990) 763.

[24] G. Källén and J. Toll, J. Math. Phys. 6 (1965) 299.

[25] K.G. Chetyrkin and F.V. Tkachov, Phys. Lett. 114B (1982) 340;

V.A. Smirnov and K.G. Chetyrkin, Theor. Math. Phys. 63 (1985) 462;

K.G. Chetyrkin and V.A. Smirnov, "The $R^{*}$ operation", Moscow State University Nuclear Physics Institute, preprint 89-3/80, Moscow 1989.

[26] K.G. Chetyrkin, A.L. Kataev and F.V. Tkachov, Nucl. Phys. B174 (1980) 345; K.G. Chetyrkin and F.V. Tkachov, Nucl. Phys. B192 (1981) 59.

[27] S. Elitzur, Institute of Advanced Study (Princeton) preprint (1979); Nucl. Phys. B212 (1983) 536.

[28] F. David, Commun. Math. Phys. 81 (1981) 149; Phys. Lett. B96 (1980) 371; Nucl. Phys. B190 (1981) 205.

[29] C. Becchi, A. Blasi, G. Bonneau, R. Collina and F. Delduc, Commun. Math. Phys. 120 (1988) 121.

[30] M.T. Grisaru, D.I. Kazakov and D. Zanon, Nucl. Phys. B287 (1987) 189. 